\title{
Global Chang's Conjecture and singular cardinals
}

\author{
Monroe Eskew ${ }^{1}$ (D) Yair Hayut ${ }^{2}$ (D)
}

Received: 31 December 2018 / Accepted: 28 January 2021 / Published online: 24 March 2021

(c) The Author(s) 2021

\begin{abstract}
We investigate the possibilities of global versions of Chang's Conjecture that involve singular cardinals. We show some ZFC limitations on such principles and prove relative to large cardinals that Chang's Conjecture can consistently hold between all pairs of limit cardinals below $\aleph_{\omega^{\omega}}$.
\end{abstract}

Keywords Chang's Conjecture $\cdot$ Scales · Diagonal Prikry forcing

Mathematics Subject Classification 03E04 - 03E10 - 03E35 - 03E55

\section{Introduction}

The Löwenheim-Skolem theorem asserts that for every pair of infinite cardinals $\kappa>\mu$ and every structure $\mathfrak{A}$ on $\kappa$ in a countable language, there is a substructure $\mathfrak{B} \subseteq \mathfrak{A}$ of size $\mu$. "Chang's Conjecture" is a type of principle strengthening this theorem to assert similar relationships between sequences of cardinals. For example $\left(\kappa_{1}, \kappa_{0}\right) \rightarrow$ $\left(\mu_{1}, \mu_{0}\right)$ says that for every structure $\mathfrak{A}$ on $\kappa_{1}$ in a countable language, there is a substructure $\mathfrak{B}$ of size $\mu_{1}$ such that $\left|\mathfrak{B} \cap \kappa_{0}\right|=\mu_{0}$. The following basic observation puts some constraints on this type of principle:

Proposition 1.1 Suppose $\kappa, \lambda \leqslant \delta$ and $\kappa^{\lambda} \geqslant \delta$. Then there is a structure $\mathfrak{A}$ on $\delta$ such that for every $\mathfrak{B} \prec \mathfrak{A}$,

$$
|\mathfrak{B} \cap \kappa|^{|\mathfrak{B} \cap \lambda|} \geqslant|\mathfrak{B} \cap \delta| .
$$

Monroe Eskew

monroe.eskew@univie.ac.at

Yair Hayut

yair.hayut@mail.huji.ac.il

1 Institut für Mathematik, Kurt Gödel Research Center, Universität Wien, Kolingasse 14-16, 1090 Wien, Austria

2 Department of Mathematics, The Hebrew University of Jerusalem, 9190401 Jerusalem, Israel 
Corollary 1.2 If $\left(\kappa_{1}, \kappa_{0}\right) \rightarrow\left(\mu_{1}, \mu_{0}\right), v \leqslant \kappa_{0}$, and $\kappa_{0}^{v} \geqslant \kappa_{1}$, then $\mu_{0}^{\min \left(\mu_{0}, v\right)} \geqslant \mu_{1}$.

From this, we immediately see that under $\mathrm{GCH},\left(\kappa^{+}, \kappa\right) \rightarrow\left(\mu^{+}, \mu\right)$ can only occur when $\operatorname{cf}(\kappa) \geqslant \operatorname{cf}(\mu)$. (The consistency of contrary cases is unknown.) This inspires the following bold conjecture:

Definition 1.3 (Global Chang's Conjecture) We say that the Global Chang's Conjecture holds if for all infinite cardinals $\mu<\kappa$ with $\operatorname{cf}(\mu) \leqslant \operatorname{cf}(\kappa),\left(\kappa^{+}, \kappa\right) \rightarrow\left(\mu^{+}, \mu\right)$.

In the paper [6], we showed, assuming the consistency of a huge cardinal, that there is a model of ZFC $+\mathrm{GCH}$ in which $\left(\kappa^{+}, \kappa\right) \rightarrow\left(\mu^{+}, \mu\right)$ holds whenever $\kappa$ is regular and $\mu<\kappa$ is infinite. Surprisingly, the full Global Chang's Conjecture is inconsistent (even without assuming GCH), as we show in Theorem 2.8. Indeed, there is a tension between instances of Chang's Conjecture at successors of singular cardinals and at double successors of singulars.

Next, we investigate other forms of Global Chang's Conjecture:

Definition 1.4 (Singular Global Chang's Conjecture) We say that the Singular Global Chang's Conjecture holds if for all infinite $\mu<\kappa$ of the same cofinality, $\left(\kappa^{+}, \kappa\right) \rightarrow$ $\left(\mu^{+}, \mu\right)$.

Obtaining the Singular Global Chang's Conjecture seems to be hard. We present here a partial result, showing that there is a model in which the Singular Global Chang's Conjecture holds for cardinals below $\aleph_{\omega^{\omega}}$.

The paper is organized as follows. In Sect. 2 we discuss some relationships between Chang's Conjecture and PCF-theoretic scales, and derive some ZFC limitations on the simultaneous occurrence of some instances of Chang's Conjecture. In Sect. 3, we introduce the technology for obtaining $\left(\aleph_{\alpha+1}, \aleph_{\alpha}\right) \rightarrow\left(\aleph_{\beta+1}, \aleph_{\beta}\right)$ for various choices of $\alpha$ and $\beta$ of countable cofinality. In Sect. 4 we construct a model in which $\left(\aleph_{\alpha+1}, \aleph_{\alpha}\right) \rightarrow\left(\aleph_{\beta+1}, \aleph_{\beta}\right)$ holds for all limit ordinals $0 \leqslant \beta<\alpha<\omega^{\omega}$. In Sect. 5, we show the consistency of $\left(\aleph_{\alpha+1}, \aleph_{\alpha}\right) \rightarrow\left(\aleph_{\beta+1}, \aleph_{\beta}\right)$ holding for a fixed $\beta$ but for $\alpha$ ranging over a longer interval of limit ordinals. We conclude with some open questions.

\section{Limitations on global Chang's Conjecture}

A useful strengthening of Chang's Conjecture is the following, introduced by Shelah [21]:

Definition 2.1 We say $\left(\kappa_{1}, \kappa_{0}\right) \rightarrow_{v}\left(\mu_{1}, \mu_{0}\right)$ if for all structures $\mathfrak{A}$ on $\kappa_{1}$ in a countable language, there is a substructure $\mathfrak{B}$ such that $|\mathfrak{B}|=\mu_{1},\left|\mathfrak{B} \cap \kappa_{0}\right|=\mu_{0}$, and $\nu \subseteq \mathfrak{B}$.

Note that nothing more is asserted by adding the subscript $v$ when $v<\omega_{1}$. These versions of Chang's Conjecture are robust under mild forcing:

Lemma 2.2 Suppose $\left(\kappa_{1}, \kappa_{0}\right) \rightarrow_{v}\left(\mu_{1}, \mu_{0}\right)$ and $\mathbb{P}$ is a $v^{+}$-c.c. partial order. Then $\Vdash_{\mathbb{P}}\left(\kappa_{1}, \kappa_{0}\right) \rightarrow_{v}\left(\mu_{1}, \mu_{0}\right)$. 
Of particular interest is the case $v=\mu_{0}$. The following lemma gives a stepping-up of the Chang's Conjecture if the distance between the cardinals considered is not too great, or enough GCH holds relatively close to the upper end. A proof is contained in [7, Section 2.2.1].

Lemma 2.3 Suppose $\left(\kappa_{1}, \kappa_{0}\right) \rightarrow_{v}\left(\mu_{1}, \mu_{0}\right)$.

(1) If $\kappa_{0}=\mu_{0}^{+v}$, then $\left(\kappa_{1}, \kappa_{0}\right) \rightarrow \mu_{0}\left(\mu_{1}, \mu_{0}\right)$.

(2) If $\lambda \leqslant \mu_{0}$ and there is $\kappa \leqslant \kappa_{0}$ such that $\kappa_{0}=\kappa^{+v}$ and $\kappa^{\lambda} \leqslant \kappa_{0}$, then $\left(\kappa_{1}, \kappa_{0}\right) \rightarrow \lambda$ $\left(\mu_{1}, \mu_{0}\right)$.

When the hypotheses of the above lemma cannot be applied, some GCH at the lower end allows a similar conclusion in a special case.

Lemma 2.4 Suppose $\mu^{<v}=\mu$, and $\left(\kappa^{+}, \kappa\right) \rightarrow\left(\mu^{+}, \mu\right)$. Then $\left(\kappa^{+}, \kappa\right) \rightarrow v\left(\mu^{+}, \mu\right)$.

Proof If $\kappa^{\nu}=\kappa$, then the conclusion follows from (2) of Lemma 2.3. Otherwise, let $\mathfrak{A}$ be a structure on $\kappa^{+}$which is isomorphic to a transitive elementary substructure of $\left(H_{\kappa^{++}}, \in, \triangleleft, \mu, v\right)$, where $\triangleleft$ is a well-order of $H_{\kappa^{++}}$. It is easy to see that the conclusion of Proposition 1.1 applies to $\mathfrak{A}$ with respect to the cardinals $\kappa, v, \kappa^{+}$. If $\mathfrak{B} \prec \mathfrak{A}$ witnesses Chang's Conjecture, then $|\mathfrak{B} \cap \kappa|^{|\mathfrak{B} \cap \nu|}=\mu^{|\mathfrak{B} \cap \nu|} \geqslant\left|\mathfrak{B} \cap \kappa^{+}\right|=\mu^{+}$. Thus $|\mathfrak{B} \cap \nu|=v$.

Let $\delta \in \mathfrak{B} \cap \nu$. Corollary 1.2 implies that $\kappa^{\delta}=\kappa$. Let $\left\langle f_{\alpha}: \alpha<\kappa\right\rangle \in \mathfrak{B}$ list all functions from $\delta$ to $\kappa$. Let $\mathfrak{B}^{\prime}=\operatorname{Hull}^{\mathfrak{A}}(\mathfrak{B} \cup \delta)$. If $\beta \in \kappa \cap \mathfrak{B}^{\prime}$, then there is function $f \in{ }^{\delta} \kappa \cap \mathfrak{B}$ and $\gamma<\delta$ such that $\beta=f(\gamma)$. Thus $\mathfrak{B}^{\prime} \cap \kappa=\left\{f_{\alpha}(\gamma): \alpha \in \mathfrak{B} \cap \kappa\right.$ and $\gamma<\delta\}$, which has size $\mu$. Now let $\mathfrak{C}=\operatorname{Hull}^{\mathfrak{A}}(\mathfrak{B} \cup v)$. Since $\mathfrak{B}$ is cofinal in $\nu$, $\mathfrak{C}=\bigcup\left\{\operatorname{Hull}^{\mathfrak{A}}(\mathfrak{B} \cup \delta): \delta \in \mathfrak{B} \cap \nu\right\}$, so $|\mathfrak{C} \cap \kappa|=\mu$.

Versions of Chang's Conjecture involving singular cardinals have a strong influence on the combinatorics in their neighborhood, even without cardinal arithmetic assumptions. Recall that if $\kappa$ is singular, a scale for $\kappa$ is a collection of functions $\left\langle f_{\alpha}: \alpha<\kappa^{+}\right\rangle$contained in some product $\prod_{i<\operatorname{cf}(\kappa)} \kappa_{i}$, where $\left\langle\kappa_{i}: i<\operatorname{cf}(\kappa)\right\rangle$ is an increasing and cofinal sequence of regular cardinals below $\kappa$, such that the functions $f_{\alpha}$ are increasing and cofinal in the partial order of the product where we put $f<g$ when $|\{i: f(i) \geqslant g(i)\}|<\operatorname{cf}(\kappa)$. It is easy to construct scales under the assumption $2^{\kappa}=\kappa^{+}$, but Shelah proved in ZFC that scales exist for all singular cardinals (see [1]).

A scale $\left\langle f_{\alpha}: \alpha<\kappa^{+}\right\rangle$is good at $\alpha$ when there is a sequence $\vec{g}=\left\langle g_{i}: i<\operatorname{cf}(\alpha)\right\rangle$ and $j_{\star}<\operatorname{cf}(\kappa)$, such that for all $j \geqslant j_{\star},\left\langle g_{i}(j): i<\operatorname{cf}(\alpha)\right\rangle$ is increasing and $\vec{g}$ and $\left\langle f_{\beta}: \beta<\alpha\right\rangle$ are interleaved (i.e., cofinal in each other). A scale is bad at $\alpha$ when it is not good at $\alpha$. A scale is better at $\alpha$ if there is a club $C \subseteq \alpha$ such that for all $\beta \in C$ there is $j<\operatorname{cf}(\kappa)$ such that $f_{\gamma}(i)<f_{\beta}(i)$ for $i \geqslant j$ and $\gamma \in C \cap \beta$. Note that if $\operatorname{cf}(\alpha)>\operatorname{cf}(\kappa)$, then being better at $\alpha$ implies being good at $\alpha$. A scale is simply called good (or better) if it is good (or better) at every $\alpha$ such that $\operatorname{cf}(\alpha)>\operatorname{cf}(\kappa)$. The key connection with Chang's Conjecture is the following (see [9] or [21]):

Lemma 2.5 If $\kappa$ is singular and $\left(\kappa^{+}, \kappa\right) \rightarrow_{\mathrm{cf}(\kappa)}\left(\mu^{+}, \mu\right)$, then there is no good scale for $\kappa$. Moreover, every scale $\left\langle f_{\alpha}: \alpha<\kappa^{+}\right\rangle$for $\kappa$ is bad at stationarily many $\alpha$ of cofinality $\mu^{+}$. 
We now show that the full Global Chang's Conjecture is inconsistent with ZFC.

Lemma 2.6 Suppose $\kappa$ is regular, $\mu<\kappa$ is singular, and $\left(\kappa^{+}, \kappa\right) \rightarrow\left(\mu^{+}, \mu\right)$. Then $\mu$ carries a better scale. Moreover, if $\mathrm{cf}(\mu)=\omega$ then $\square_{\mu}^{*}$ holds.

Proof Let us start with a general observation, following [8, Theorem 2.15].

Claim 2.7 Let $\mu<\kappa=\operatorname{cf}(\kappa)$ be cardinals. Let $\theta$ be a regular cardinal above $\kappa^{+}$. If $H$ is the transitive collapse of some elementary substructure of $H_{\theta}$ of size $\kappa^{+}$ containing $\kappa^{+}$, and $M \prec H$ is such that $\left|M \cap \kappa^{+}\right|=\mu^{+}$and $|M \cap \kappa|=\mu$, then $\operatorname{cf}(\sup (M \cap \kappa))=\operatorname{cf}(\mu)$.

Proof Fix in such an $H$ a sequence $\left\langle x_{\alpha}: \alpha<\kappa^{+}\right\rangle$of "strongly almost disjoint" unbounded subsets of $\kappa$. That is, for every $\alpha<\kappa^{+}$, there is a sequence $\left\langle\gamma_{\beta}^{\alpha}: \beta<\alpha\right\rangle \in$ $H$ of ordinals below $\kappa$ such that $\left\langle x_{\beta} \backslash \gamma_{\beta}^{\alpha}: \beta<\alpha\right\rangle$ is pairwise disjoint. This principle, due to Shelah, is called $\mathrm{ADS}_{\kappa}$ and it holds for $\kappa$ regular (see [4,22]).

Let $M \prec H$ be as above. Let $f: \mu \rightarrow M \cap \kappa$ be a bijection. If $\operatorname{cf}(\sup (M \cap \kappa)) \neq$ $\operatorname{cf}(\mu)$, then for each $\alpha<M \cap \kappa^{+}$there is $\delta_{\alpha}<\mu$ such that $f\left[\delta_{\alpha}\right] \cap x_{\alpha}$ is cofinal in $M \cap \kappa$. Since $\left|M \cap \kappa^{+}\right|=\mu^{+}$, there is a set $Y \subseteq M \cap \kappa^{+}$of size $\mu^{+}$and a fixed $\delta<\mu$ such that $\delta_{\alpha}=\delta$ for all $\alpha \in Y$. Let $\zeta \in M \cap \kappa^{+}$be large enough so that $|Y \cap \zeta|=\mu$. Note that $\left\langle\gamma_{\beta}^{\zeta}: \beta<\zeta\right\rangle \in M$ and thus for every $\beta \in M \cap \zeta, \gamma_{\beta}^{\zeta} \in M \cap \kappa$.

For $\beta \in Y \cap \zeta$, let $y_{\beta}=f[\delta] \cap x_{\beta} \backslash \gamma_{\beta}^{\zeta}$. Then $\left\{f^{-1}\left[y_{\beta}\right]: \beta \in Y \cap \alpha\right\}$ is a collection of $\mu$-many pairwise disjoint subsets of $\delta$, which is impossible.

Let us return to the proof of the lemma.

By a theorem of Shelah [21], $\kappa$ carries a "partial weak square", a weak square sequence that misses only cofinality $\kappa$. That is, there is a sequence $\left\langle\mathfrak{C}_{\alpha}: \alpha<\kappa^{+}\right\rangle$such that whenever $\omega \leqslant \operatorname{cf}(\alpha)<\kappa$, then $\bigodot_{\alpha}$ is a nonempty collection of size $\leqslant \kappa$ such that each $\mathcal{C} \in \mathcal{C}_{\alpha}$ is a club subset of $\alpha$ of size $<\kappa$, and if $\mathcal{C} \in \mathcal{C}_{\alpha}$ and $\beta \in \lim \mathcal{C}$, then $\mathcal{C} \cap \beta \in \mathcal{C}_{\beta}$.

Let $M \prec H$ be as above, with $\overrightarrow{\mathcal{C}} \in M$ a partial weak square at $\kappa$. Let $\pi: M \rightarrow N$ be the transitive collapse. Let $\overrightarrow{\mathcal{D}}=\pi(\overrightarrow{\mathcal{C}})$. Since ot $\left(M \cap \kappa^{+}\right)=\mu^{+}$and $\left|M \cap \mathcal{C}_{\alpha}\right| \leqslant \mu$ for each $\alpha \in M \cap \kappa^{+}, \overrightarrow{\mathcal{D}}$ is a sequence $\left\langle\mathcal{D}_{\alpha}: \alpha<\mu^{+}\right\rangle$, such that each $\mathcal{D}_{\alpha}$ has size $\leqslant \mu$, if $\mathcal{D} \in \mathcal{D}_{\alpha}$ and $\beta \in \lim \mathcal{D}$, then $\mathcal{D} \cap \beta \in \mathcal{D}_{\beta}$, and $\mathcal{D}_{\alpha}$ is nonempty whenever $\alpha$ is a limit ordinal such that $\mathrm{cf}\left(\pi^{-1}(\alpha)\right) \neq \kappa$. If $\alpha$ is such that $\operatorname{cf}\left(\pi^{-1}(\alpha)\right)=\kappa$, then there is an increasing cofinal map $f: \kappa \rightarrow \pi^{-1}(\alpha)$ in $M$, which implies that $\operatorname{cf}(\alpha)=\operatorname{cf}(\mu)$. Therefore, $\mathcal{D}_{\alpha}$ is nonempty whenever $\operatorname{cf}(\alpha) \neq \operatorname{cf}(\mu)$. Furthermore, if $\mathcal{D} \in \mathcal{D}_{\alpha}$, then ot $(D)<\pi(\kappa)$.

Next, we modify $\overrightarrow{\mathcal{D}}$ to a sequence $\overrightarrow{\mathcal{E}}$ with the same properties except that $|\mathcal{C}|<\mu$ whenever $\mathcal{C} \in \mathcal{E}_{\alpha}$ and $\alpha<\mu^{+}$. It is easy to show by induction that for each $\eta<\mu^{+}$, there is a "short square" of length $\eta$-a coherent sequence of clubs $\left\langle E_{\alpha}: \alpha<\eta\right\rangle$ such that $\left|E_{\alpha}\right|<\mu$ for each $\alpha<\eta$. Fix such a sequence $\left\langle E_{\alpha}: \alpha<\pi(\kappa)\right\rangle$. For each $\alpha<\mu^{+}$, let $\mathcal{E}_{\alpha}=\left\{\left\{\beta \in D: \operatorname{ot}(D \cap \beta) \in E_{\mathrm{ot}(D)}\right\}: D \in \mathcal{D}_{\alpha}\right\}$. Clearly each element of each $\mathcal{E}_{\alpha}$ has size $<\mu$. If $C \in \mathcal{E}_{\alpha}$ and $\beta \in \lim C$, then there is $D \in \mathcal{D}_{\alpha}$ such that $\beta \in \lim D$ and $C=\left\{\beta \in D: \operatorname{ot}(D \cap \beta) \in E_{\mathrm{ot}(D)}\right\}$. Thus $D \cap \beta \in \mathcal{D}_{\beta}$ and ot $(D \cap \beta) \in \lim E_{\mathrm{ot}(D)}$, so $C \cap \beta \in \mathcal{E}_{\beta}$. 
Note that $\overrightarrow{\mathcal{E}}$ is a partial weak square, avoiding only ordinals of cofinality $\operatorname{cf}(\mu)$. Thus if $\operatorname{cf}(\mu)=\omega$, one can easily obtain a weak square sequence by completing the missing points in $\overrightarrow{\mathcal{E}}$.

Fix a scale for $\mu,\left\langle f_{\alpha}: \alpha<\mu^{+}\right\rangle \subseteq \prod_{i<\operatorname{cf}(\mu)} \mu_{i}$. Let us inductively construct a better scale $\left\langle g_{\alpha}: \alpha<\mu^{+}\right\rangle$as follows. Let $g_{0}=f_{0}$. If $\mathcal{E}_{\alpha}$ is empty, let $g_{\alpha}=f_{\gamma}$, where $\gamma \geqslant \alpha$ and $f_{\gamma}$ eventually dominates $g_{\beta}$ for each $\beta<\alpha$. If $\mathcal{E}_{\alpha}$ is nonempty, first, for all $C \in \mathcal{E}_{\alpha}$, define

$$
g_{C}(i)= \begin{cases}\sup \left\{g_{\beta}(i)+1: \beta \in C\right\} & \text { if } \mu_{i}>|C| \\ 0 & \text { otherwise }\end{cases}
$$

Then let $g_{\alpha}=f_{\gamma}$, where $\gamma \geqslant \alpha$ and $f_{\gamma}$ eventually dominates $g_{\beta}$ for each $\beta<\alpha$ and $g_{C}$ for each $C \in \mathcal{E}_{\alpha}$.

Clearly $\left\langle g_{\alpha}: \alpha<\mu^{+}\right\rangle$is a scale. To check betterness, if $\operatorname{cf}(\alpha)>\operatorname{cf}(\mu)$, let $C \in \mathcal{E}_{\alpha}$. If $\beta \in \lim C$, then $C \cap \beta \in \mathcal{E}_{\beta}$. There is $i<\operatorname{cf}(\mu)$ such that $g_{C \cap \beta}(j)>g_{\gamma}(j)$ for $i<j<\operatorname{cf}(\mu)$ and $\gamma \in C \cap \beta$. Thus if $C^{\prime}$ is the set of limit points of some $C \in \mathcal{E}_{\alpha}$, then for all $\beta \in C^{\prime}$ there is $i<\operatorname{cf}(\mu)$ such that $g_{\beta}(j)>g_{\gamma}(j)$ for $i<j<\operatorname{cf}(\mu)$ and $\gamma \in C^{\prime} \cap \beta$.

Combining the above with Lemmas 2.4 and 2.5, we have:

Theorem 2.8 Suppose $\kappa$ is singular, $\lambda>\kappa$ is regular, $\left(\lambda^{+}, \lambda\right) \rightarrow\left(\kappa^{+}, \kappa\right)$, and $\operatorname{cf}(\kappa) \leqslant \mu<\kappa$. Then $\left(\kappa^{+}, \kappa\right) \not \rightarrow \operatorname{cf}(\kappa)\left(\mu^{+}, \mu\right)$. Thus if $\mu^{<\operatorname{cf}(\kappa)}=\mu$, then $\left(\kappa^{+}, \kappa\right) \not \nrightarrow$ $\left(\mu^{+}, \mu\right)$.

Corollary $2.9\left[\aleph_{0}, \aleph_{\omega}\right]$ is the maximal initial interval of cardinals on which the Global Chang's Conjecture can hold.

The negative direction follows from Theorem 2.8 and the positive direction is proven in [6, Section 5].

It seems to be unknown whether $\left(\kappa^{+}, \kappa\right) \rightarrow\left(\mu^{+}, \mu\right)$ is equivalent to $\left(\kappa^{+}, \kappa\right) \rightarrow \mu$ $\left(\mu^{+}, \mu\right)$ for regular $\mu$. However, further analysis of scales allows us to rule out some instances of Chang's Conjecture in ZFC, and to show that these two notions are not in general equivalent for singular $\mu$, even under $\mathrm{GCH}$. The authors are grateful to Chris Lambie-Hanson for showing us how to prove the following:

Theorem 2.10 Suppose $\kappa$ is a singular cardinal and $\vec{f}=\left\langle f_{\alpha}: \alpha<\kappa^{+}\right\rangle$is a scale for $\kappa$. There is a club $C \subseteq \kappa^{+}$such that for all regular cardinals $\mu, v$ such that $\operatorname{cf}(\kappa)<\mu<\mu^{+3} \leqslant \nu<\mu^{+\operatorname{cf}(\kappa)} \leqslant \kappa, \vec{f}$ is good at every $\alpha \in C$ of cofinality $\nu$.

Proof Suppose $\operatorname{cf}(\kappa)<\mu<\mu^{+3} \leqslant v<\mu^{+\operatorname{cf}(\kappa)} \leqslant \kappa$. By [1, Theorem 2.21], there is a club $C_{\mu, \nu} \subseteq \kappa^{+}$such that for every $\alpha \in C_{\mu, \nu}$ of cofinality $v,\left\langle f_{\beta}: \beta<\alpha\right\rangle$ has an exact upper bound $g$ such that $\operatorname{cf}(g(i)) \geqslant \mu$ for all $i$. $g$ being an exact upper bound means that $g$ is an upper bound to $\left\langle f_{\beta}: \beta<\alpha\right\rangle$, and for every $h<g$, there is $\beta<\alpha$ such that $h<f_{\beta}$.

The arguments for [17, Lemmas 6-8] show that $\operatorname{cf}(g(i))=v$ on a cobounded set of $i<\operatorname{cf}(\kappa)$, which implies $\vec{f}$ is good at $\alpha$. For the reader's convenience: Let $\left\langle\alpha_{j}: j<v\right\rangle$ be cofinal in $\alpha$. We cannot have that $\operatorname{cf}(g(i))>v$ for all $i$ in an unbounded set 
$X \subseteq \operatorname{cf}(\kappa)$. For then there would be an $i^{*}<\operatorname{cf}(\kappa)$, an unbounded $Y \subseteq \nu$, and an $h<g$ such that $f_{\alpha_{j}}(i)<h(i)<g(i)$ for $i \in X \backslash i^{*}$ and $j \in Y$, contradicting that $g$ is an exact upper bound. Thus there is some $\nu^{\prime} \in[\mu, \nu]$ and an unbounded $X \subseteq \operatorname{cf}(\kappa)$ such that $\operatorname{cf}(g(i))=v^{\prime}$ for all $i \in X$. Let $\left\langle g_{k}: k<v^{\prime}\right\rangle$ be a pointwise increasing sequence such that $\sup _{k<v^{\prime}} g_{k}(i)=g(i)$ for all $i \in X$. Since $g$ is an exact upper bound, for each $k<v^{\prime}$, there is $j<v$ such that $g_{k}\left\lceil X<f_{\alpha_{j}}\lceil X\right.$. Also, for each $j<v$, there is $i^{*}<\operatorname{cf}(\kappa)$ such that $f_{\alpha_{j}}(i)<g(i)$ for $i \in X \backslash i^{*}$, and thus some $k<v^{\prime}$ such that $f_{\alpha_{j}} \uparrow X<g_{k}\left\lceil X\right.$. This implies $\nu^{\prime}=v$.

Finally, we can take the intersection of all the $C_{\nu, \mu}$ for regular $\nu, \mu<\kappa^{+}$to get the desired club $C$.

Therefore, if $\kappa$ is singular, $\left(\kappa^{+}, \kappa\right) \rightarrow_{\mathrm{cf}(\kappa)}\left(\mu^{+}, \mu\right)$ fails whenever $\mathrm{cf}(\kappa)^{+3} \leqslant \mu<$ $\operatorname{cf}(\kappa)^{+\mathrm{cf}(\kappa)}$. However, it is possible that the version of Chang's Conjecture holds when we drop the subscript "cf $(\kappa)$ " on the arrow:

Proposition 2.11 Suppose there is a 3-huge cardinal. Then there are singular cardinals $\lambda<\delta$ such that $\operatorname{cf}(\delta)<\lambda<\operatorname{cf}(\delta)^{+\operatorname{cf}(\delta)}$ and $\left(\delta^{+}, \delta\right) \rightarrow\left(\lambda^{+}, \lambda\right)$.

Proof Let $j: V \rightarrow M$ have critical point $\kappa$, with $M^{j^{3}(\kappa)} \subseteq M$. Let $\delta=j^{2}(\kappa)^{+j(\kappa)}$ and let $\lambda=j(\kappa)^{+\kappa}$. Let $\mathfrak{A}$ be any structure on $\delta^{+}$. In $M, j[\mathfrak{A}] \prec j(\mathfrak{A})$, and we have that $|j[\mathfrak{A}]|=\delta^{+}$and $|j[\mathfrak{A}] \cap j(\delta)|=\delta$. Reflecting through $j$, we have that there is $\mathfrak{B} \prec \mathfrak{A}$ such that $|\mathfrak{B}|=j(\kappa)^{+\kappa+1}=\lambda^{+}$and $|\mathfrak{B} \cap \delta|=j(\kappa)^{+\kappa}=\lambda$.

\section{Chang's Conjecture between successors of various singulars}

Recall that a partial order is $(\kappa, \lambda)$-distributive if forcing with it adds no functions from $\kappa$ to $\lambda$. The following lemma is a mild generalization of a lemma that was proved in $[6]$.

Lemma 3.1 Let $\gamma<\kappa$ be such that $\kappa^{+\gamma}$ is a strong limit cardinal and $\kappa$ is $\kappa^{+\gamma+1}$. supercompact, as witnessed by an embedding $j: V \rightarrow M$. If $\mathcal{U}$ is the ultrafilter on $\kappa$ derived from $j$, then there is $A \in \mathcal{U}$ such that for every $\alpha<\beta$ in $A \cup\{\kappa\}$ and every iteration $\mathbb{P} * \dot{\mathbb{Q}}$ of size $<\beta^{+\gamma}$, such that $\mathbb{P}$ is $\alpha^{+\gamma+1}$-Knaster and $\Perp_{\mathbb{P}} \dot{\mathbb{Q}}$ is $\left(\alpha^{+\gamma+1}, \alpha^{+\gamma+1}\right)$-distributive,

$$
\Vdash_{\mathbb{P} * \dot{\mathbb{Q}}}\left(\beta^{+\gamma+1}, \beta^{+\gamma}\right) \rightarrow_{\alpha^{+\gamma}}\left(\alpha^{+\gamma+1}, \alpha^{+\gamma}\right) .
$$

Proof We show that for a set $A \in \mathcal{U}$, for every $\alpha \in A$ and every iteration $\mathbb{P} * \dot{\mathbb{Q}}$ satisfying the hypothesis for $\beta=\kappa$ forces $\left(\kappa^{+\gamma+1}, \kappa^{+\gamma}\right) \rightarrow \alpha^{+\gamma}\left(\alpha^{+\gamma+1}, \alpha^{+\gamma}\right)$. Then standard reflection arguments yield the desired conclusion. By Lemma 2.3, it suffices to prove that for all $\alpha \in A$, every such $\mathbb{P} * \dot{\mathbb{Q}}$ forces $\left(\kappa^{+\gamma+1}, \kappa^{+\gamma}\right) \rightarrow \gamma\left(\alpha^{+\gamma+1}, \alpha^{+\gamma}\right)$, since by the assumptions that $\kappa^{+\gamma}$ is a strong limit and $|\mathbb{P} * \dot{\mathbb{Q}}|<\kappa^{+\gamma}$, it is forced that for some $\lambda \in\left[\kappa, \kappa^{+\gamma}\right), \lambda^{\kappa}<\kappa^{+\gamma}$, so we may increase the subscript to $\alpha^{+\gamma}$. If the claim fails, then on a set $B \in \mathcal{U}$, for every $\alpha \in B$, there is an iteration $\mathbb{P}_{\alpha} * \dot{\mathbb{Q}}_{\alpha}$ and a name for a function $\dot{f}_{\alpha}:\left(\kappa^{+\gamma+1}\right)^{<\omega} \rightarrow \kappa^{+\gamma}$ such that it is forced that for every $X \subseteq \kappa^{+\gamma+1}$ of size $\alpha^{+\gamma+1}$ with $\gamma \subseteq X$, the closure of $X$ under $\dot{f}_{\alpha}$ contains $\alpha^{+\gamma+1}$-many ordinals below $\kappa^{+\gamma}$. We may assume that $\dot{f}_{\alpha}$ is forced to be closed under compositions. 
In $M$, let $\mathbb{P} * \dot{\mathbb{Q}}=j\left(\left\langle\mathbb{P}_{\alpha} * \dot{\mathbb{Q}}_{\alpha}: \alpha<\kappa\right\rangle\right)(\kappa)$ and let $\dot{f}=j\left(\left\langle\dot{f}_{\alpha}: \alpha<\kappa\right\rangle\right)(\kappa)$. Let $X=j\left[\kappa^{+\gamma+1}\right]$. Note that $X$ is a subset of $j\left(\kappa^{+\gamma+1}\right)$ containing $\gamma$ and of size $\kappa^{+\gamma+1}$. By hypothesis, $\Vdash_{\mathbb{P} * \dot{\mathbb{Q}}}^{M}\left|\dot{f}\left[X^{<\omega}\right]\right|=\kappa^{+\gamma+1}$. Since $j\left(\kappa^{+\gamma}\right)$ is singular, it is forced that there is a sequence $\left\langle\dot{b}_{\alpha}: \alpha<\kappa^{+\gamma+1}\right\rangle \subseteq X$ such that $\left\langle\dot{f}\left(\dot{b}_{\alpha}\right): \alpha<\kappa^{\gamma+1}\right\rangle$ is a strictly increasing sequence of ordinals below $j\left(\kappa^{+\xi}\right)$, for some $\xi<\gamma$. Let $v<\gamma$ and $\left(p_{0}, \dot{q}_{0}\right) \in \mathbb{P} * \dot{\mathbb{Q}}$ be such that $|\mathbb{P} * \dot{\mathbb{Q}}|<j\left(\kappa^{+v}\right)$ and $\left(p_{0}, \dot{q}_{0}\right) \Vdash \dot{f}\left(\dot{b}_{\alpha}\right)<j\left(\kappa^{+v}\right)$ for all $\alpha<\kappa^{+\gamma+1}$.

Since $\dot{\mathbb{Q}}$ adds no subsets to $X$, there is a $\mathbb{P}$-name $\dot{Y}$ and a condition $\left(p_{1}, \dot{q}_{1}\right) \leqslant\left(p_{0}, \dot{q}_{0}\right)$ such that $\left(p_{1}, \dot{q}_{1}\right) \Vdash\left\langle\dot{b}_{\alpha}: \alpha<\kappa^{+\gamma+1}\right\rangle=\dot{Y}$. Next, for each $\alpha<\kappa^{+\gamma+1}$, find $r_{\alpha} \leqslant p_{1}$ and $a_{\alpha} \in\left(\kappa^{+\gamma+1}\right)^{<\omega}$ such that $r_{\alpha} \Vdash_{\mathbb{P}} j\left(\check{a}_{\alpha}\right)=\dot{Y}(\alpha)$. Since $\mathbb{P}$ is $\kappa^{+\gamma+1}$-Knaster, there is $Z \subseteq \kappa^{+\gamma+1}$ of size $\kappa^{+\gamma+1}$ such that $r_{\alpha}$ and $r_{\beta}$ are compatible for $\alpha, \beta \in Z$. Therefore, for $\alpha<\beta$ in $Z$, there is $r \in \mathbb{P}$ such that $\left(r, \dot{q}_{1}\right) \Vdash \dot{f}\left(j\left(\check{a}_{\alpha}\right)\right)<\dot{f}\left(j\left(\check{a}_{\beta}\right)\right)<$ $j\left(\kappa^{+v}\right)$.

Reflecting these statements to $V$, we have that for $\alpha<\beta$ in $Z$, there are $\gamma<\kappa$ and $(p, \dot{q}) \in P_{\gamma} * \dot{\mathbb{Q}}_{\gamma}$ such that $\left|\mathbb{P}_{\gamma} * \dot{\mathbb{Q}}_{\gamma}\right|<\kappa^{+v}$ and $(p, \dot{q}) \Vdash_{\mathbb{P}_{\gamma} * \dot{\mathbb{Q}}_{\gamma}}^{V} \dot{f}_{\gamma}\left(\check{a}_{\alpha}\right)<\dot{f}_{\gamma}\left(\check{a}_{\beta}\right)<$ $\kappa^{+v}$. This defines a coloring of $\left[\kappa^{+\gamma+1}\right]^{2}$ in $\kappa^{+\nu}$-many colors. Since $\kappa^{+\gamma}$ is a strong limit, the Erdős-Rado Theorem implies that there is a set $H \subseteq Z$ of size $\kappa^{+v+1}$ such that all pairs in $[H]^{2}$ get the same color. Thus we have a fixed $\eta$ and a fixed $(p, \dot{q}) \in \mathbb{P}_{\eta} * \dot{\mathbb{Q}}_{\eta}$ such that $(p, \dot{q}) \Vdash \dot{f}_{\eta}\left(a_{\alpha}\right)<\dot{f}_{\eta}\left(a_{\beta}\right)<\kappa^{+v}$ for $\alpha<\beta$ in $H$. This is a contradiction.

Corollary 3.2 If there is a $(+\omega+1)$-supercompact cardinal, then there is a forcing extension in which $\left(\aleph_{\alpha+1}, \aleph_{\alpha}\right) \rightarrow\left(\aleph_{\beta+1}, \aleph_{\beta}\right)$ holds for all limit ordinals $0 \leqslant \beta<$ $\alpha<\omega^{2}$.

Proof Let $\kappa$ be $\kappa^{+\omega+1}$-supercompact, and let $A \subseteq \kappa$ be given by Lemma 3.1. Let $\left\langle\alpha_{i}: i<\omega\right\rangle$ enumerate the first $\omega$ elements of $A$. Let

$$
\mathbb{P}=\operatorname{Col}\left(\omega, \alpha_{0}^{+\omega}\right) \times \prod_{n<\omega} \operatorname{Col}\left(\alpha_{n}^{+\omega+2}, \alpha_{n+1}\right)
$$

Clearly, $\mathbb{P}$ forces that $\alpha_{n}^{+\omega}=\aleph_{\omega \cdot n}$ for all $n$. For a fixed $n$, we can factor $\mathbb{P}$ as $\mathbb{Q}_{0} \times \operatorname{Col}\left(\alpha_{n}^{+\omega+2}, \alpha_{n+1}\right) \times \mathbb{Q}_{1}$. By Lemma 3.1, the product of the first two factors forces $\left(\alpha_{n+1}^{+\omega+1}, \alpha_{n+1}^{+\omega}\right) \rightarrow \alpha_{n}^{+\omega}\left(\alpha_{n}^{+\omega+1}, \alpha_{n}^{+\omega}\right)$. Since $\mathbb{Q}_{1}$ remains $\alpha_{n+1}^{+\omega+2}$-distributive after this, the instance of Chang's Conjecture is preserved. Since Chang's Conjecture is transitive, i.e., $\left(\kappa_{1}, \kappa_{0}\right) \rightarrow\left(\mu_{1}, \mu_{0}\right)$ and $\left(\mu_{1}, \mu_{0}\right) \rightarrow\left(v_{1}, v_{0}\right)$ implies $\left(\kappa_{1}, \kappa_{0}\right) \rightarrow\left(v_{1}, v_{0}\right)$, the conclusion follows.

The limitation of our argument so far is that we only get Chang's Conjecture between successors of singulars for which there are tail-end sequences of cardinals below that are order-isomorphic. We will overcome this with a forcing that collapses singular cardinals onto others of different types while preserving their successors and the desired instances of Chang's Conjecture.

Theorem 3.3 Assume GCH. Suppose $\alpha<\beta$ are countable limit ordinals and $\kappa$ is $\kappa^{+\beta+1}$-supercompact. Then there is a forcing extension in which $\left(\aleph_{\beta+1}, \aleph_{\beta}\right) \rightarrow$ $\left(\aleph_{\alpha+1}, \aleph_{\alpha}\right)$. 
The proof breaks into cases depending on the "tail types" of $\alpha$ and $\beta$. For ordinals $\alpha \geqslant \beta$, let $\alpha-\beta$ be the unique $\gamma$ such that $\alpha=\beta+\gamma$. For an ordinal $\alpha$, let $\tau(\alpha)$ (the tail of $\alpha$ ) be $\min _{\beta<\alpha}(\alpha-\beta)$. Let $\iota(\alpha)$ be the least $\beta$ such that $\alpha=\beta+\tau(\alpha)$. An ordinal $\alpha$ is indecomposable iff $\alpha=\tau(\alpha)$, and all tails are indecomposable.

Case 1: $\tau(\alpha)=\tau(\beta)=\gamma$, or $\alpha=0$. Note that $\iota(\beta) \geqslant \alpha$, and let $\delta=\iota(\beta)-\alpha$. Let $A \subseteq \kappa$ be given by Lemma 3.1 (with respect to $\gamma$ ). Let $\zeta<\eta$ be in $A$, and force with $\operatorname{Col}\left(\zeta^{+\gamma+\delta+2}, \eta\right)$, so that the ordertype of the set of cardinals between $\zeta^{+\gamma}$ and $\eta^{+\gamma}$ becomes $\delta+\gamma$. By Lemma 3.1, we have $\left(\eta^{+\gamma+1}, \eta^{+\gamma}\right) \rightarrow_{\zeta^{+\gamma}}\left(\zeta^{+\gamma+1}, \zeta^{+\gamma}\right)$. If $\alpha=0$, force with $\operatorname{Col}\left(\omega, \zeta^{+\gamma}\right)$, and if $\alpha>0$, force with $\operatorname{Col}\left(\aleph_{\iota}(\alpha)+1, \zeta\right)$. In both cases, Chang's Conjecture is preserved, and we get $\left|\zeta^{+\gamma}\right|=\aleph_{\alpha}$ and $\eta^{+\gamma}=\aleph_{\alpha+\delta+\gamma}=\aleph_{\beta}$.

For the other cases, we will use a variation on the Gitik-Sharon forcing [12], which singularlizes a large cardinal while collapsing a singular cardinal above it. The following definition is standard (see [11]).

Definition 3.4 A structure $\left\langle\mathbb{P}, \leqslant, \leqslant^{*}\right\rangle$ is a Prikry-type forcing when $\leqslant$ and $\leqslant *$ are partial orders of $\mathbb{P}$ (called extension and direct extension respectively), with $p \leqslant{ }^{*} q \Rightarrow$ $p \leqslant q$, and such that whenever $\sigma$ is a statement in the forcing language of $\langle\mathbb{P}, \leqslant\rangle$ and $p \in \mathbb{P}$, then there is $q \leqslant{ }^{*} p$ deciding $\sigma$. Such a forcing is called weakly $\kappa$-closed for a cardinal $\kappa$ if $\left\langle\mathbb{P}, \leqslant^{*}\right\rangle$ is $\kappa$-closed.

It is easy to see that if $\mathbb{P}$ is of Prikry type and weakly $\kappa^{+}$-closed, then it is $(\kappa, \kappa)$ distributive.

Suppose $\gamma<\delta$ are limit ordinals of countable cofinality, and $\vec{\gamma}=\left\langle\gamma_{i}: 1 \leqslant i<\omega\right\rangle$, $\vec{\delta}=\left\langle\delta_{i}: 1 \leqslant i<\omega\right\rangle$ are sequences such that:

(1) $\vec{\gamma}$ is strictly increasing with $\sup _{i} \gamma_{i}=\gamma$.

(2) $\vec{\delta}$ is nondecreasing with $\gamma \leqslant \delta_{1}$ and $\sum_{i} \delta_{i}=\delta$.

Suppose $\kappa>\delta$ is $\kappa^{+\gamma_{n}}$-supercompact for each $n \geqslant 1$, and $\mu<\kappa$ is regular. For $1 \leqslant n<\omega$, let $U_{n}$ be a $\kappa$-complete normal measure on $\mathcal{P}_{\kappa}\left(\kappa^{+\gamma_{n}}\right)$, and let $j_{n}: V \rightarrow$ $M_{n} \cong \operatorname{Ult}\left(V, U_{n}\right)$ be the ultrapower embedding. By the closure of the ultrapowers and $\mathrm{GCH}$, we may choose an $M_{n}$-generic $K_{n} \subseteq \operatorname{Col}\left(\kappa^{+\delta_{n}+2}, j_{n}(\kappa)\right)^{M_{n}}$. Let $\vec{U}=$ $\left\langle U_{n}: n<\omega\right\rangle$ and $\vec{K}=\left\langle K_{n}: n<\omega\right\rangle$.

With these choices made, we may define the forcing $\mathbb{P}(\mu, \vec{\gamma}, \vec{\delta}, \vec{U}, \vec{K})$, which will have the following properties:

- The forcing is of Prikry type, weakly $\mu$-closed, and $\kappa^{+\gamma}$-centered (and thus has the $\kappa^{+\gamma+1}$-c.c.).

- $\kappa$ is forced to become $\mu^{+\delta}$.

- $\left(\kappa^{+\gamma}\right)^{V}$ is collapsed to $\kappa$.

Conditions in $\mathbb{P}(\mu, \vec{\gamma}, \vec{\delta}, \vec{U}, \vec{K})$ are sequences

$$
\left\langle f_{0}, x_{1}, f_{1}, \ldots, x_{n}, f_{n}, F_{n+1}, F_{n+2}, \ldots\right\rangle
$$

where:

(1) For $1 \leqslant i \leqslant n, x_{i} \in \mathcal{P}_{\kappa}\left(\kappa^{+\gamma_{i}}\right)$, and $\kappa_{i}:=x_{i} \cap \kappa$ is inaccessible.

(2) For $1 \leqslant i<n, x_{i} \subseteq x_{i+1}$, and $\kappa_{i+1}>\left|x_{i}\right|$. 
(3) $f_{0} \in \operatorname{Col}(\mu, \kappa)$, and $\operatorname{ran}\left(f_{0}\right) \subseteq \kappa_{1}$ if $x_{1}$ is defined.

(4) For $1 \leqslant i<n, f_{i} \in \operatorname{Col}\left(\kappa_{i}^{+\delta_{i}+2}, \kappa_{i+1}\right)$.

(5) $f_{n} \in \operatorname{Col}\left(\kappa_{n}^{+\delta_{n}+2}, \kappa\right)$.

(6) For $i>n$, $\operatorname{dom} F_{i} \in U_{i}$.

(7) For $i>n$ and $x \in \operatorname{dom} F_{i}, x \supseteq x_{n}$ and $\kappa_{x}:=x \cap \kappa$ is an inaccessible cardinal greater than $\left|x_{n}\right|+\sup \left(\operatorname{ran}\left(f_{n}\right)\right)$.

(8) For $i>n$ and $x \in \operatorname{dom} F_{i}, F_{i}(x) \in \operatorname{Col}\left(\kappa_{x}^{+\delta_{i}+2}, \kappa\right)$.

(9) For $i>n,\left[F_{i}\right]_{U_{i}} \in K_{i}$.

Suppose $p=\left\langle f_{0}, \ldots, x_{n}, f_{n}, F_{n+1}, \ldots\right\rangle$ and $q=\left\langle f_{0}^{\prime}, \ldots, x_{m}^{\prime}, f_{m}^{\prime}, F_{m+1}^{\prime}, \ldots\right\rangle$. We say $q \leqslant p$ when:

(1) $m \geqslant n$.

(2) $f_{i}^{\prime} \supseteq f_{i}$ for $i \leqslant n$, and $x_{i}=x_{i}^{\prime}$ for $1 \leqslant i \leqslant n$.

(3) For $n<i \leqslant m, x_{i}^{\prime} \in \operatorname{dom} F_{i}$ and $f_{i}^{\prime} \supseteq F_{i}\left(x_{i}^{\prime}\right)$.

(4) For $i>m$, $\operatorname{dom} F_{i}^{\prime} \subseteq \operatorname{dom} F_{i}$, and $F_{i}^{\prime}(x) \supseteq F_{i}(x)$ for $x \in \operatorname{dom} F_{i}^{\prime}$.

For $p$ as above, let $\operatorname{stem}(p)=\left\langle f_{0}, \ldots, x_{n}, f_{n}\right\rangle$, and say the length of $p$ is $n$. (The stem of a length- 0 condition is of the form $\left\langle f_{0}\right\rangle$.)

Lemma 3.5 Suppose $\mu, \vec{\gamma}, \vec{\delta}, \vec{U}, \vec{K}$ are as above, and $p=\left\langle f_{0}, x_{1}, \ldots, x_{n}, f_{n}\right\rangle-\vec{F}$ is a condition of length $n>0$. Then $\mathbb{P}(\mu, \vec{\gamma}, \vec{\delta}, \vec{U}, \vec{K})\lceil p$ is canonically isomorphic to

$$
\begin{aligned}
\operatorname{Col}\left(\mu, \kappa_{1}\right) \uparrow & f_{0} \times \cdots \times \operatorname{Col}\left(\kappa_{n-1}^{+\delta_{n-1}+2}, \kappa_{n}\right) \\
& \uparrow f_{n-1} \times \mathbb{P}\left(\kappa_{n}^{+\delta_{n}+2}, \vec{\gamma}^{\prime}, \vec{\delta}^{\prime}, \vec{U}^{\prime}, \vec{K}^{\prime}\right) \uparrow\left\langle f_{n}\right\rangle \vec{F}^{\prime},
\end{aligned}
$$

where for each sequence $s \in\{\vec{\gamma}, \vec{\delta}, \vec{U}, \vec{K}, \vec{F}\}, s^{\prime}$ is the sequence such that $s^{\prime}(m)=$ $s(n+m)$ for $m \geqslant 1$.

We say $q \leqslant{ }^{*} p$ when $q \leqslant p$ and they have the same length. If $q \leqslant p$ and $\operatorname{stem}(p)$ is an initial segment of $\operatorname{stem}(q)$, we say $q$ is an end-extension of $p$, or $q \preceq p$. Given a sequence $\vec{F}=\left\langle F_{i}: 1 \leqslant i<\omega\right\rangle$ such that $\langle\varnothing\rangle-\vec{F}$ is a condition of length 0 , and another condition $p=\operatorname{stem}(p) \frown\left\langle H_{i}: n<i<\omega\right\rangle$, define

$$
\begin{aligned}
p \wedge \vec{F}:=\operatorname{stem}(p) & -\left\{\left\langle\left\langle x, F_{i}(x) \cup H_{i}(x)\right\rangle: x \in \operatorname{dom} F_{i} \cap \operatorname{dom} H_{i}\right.\right. \\
& \text { and } \left.\left.F_{i}(x) \cup H_{i}(x) \text { is a function }\right\}: n<i<\omega\right\rangle .
\end{aligned}
$$

Note that $p \wedge \vec{F}$ is both $\preceq$ and $\leqslant{ }^{*} p$, but $p \wedge \vec{F}$ is not necessarily $\leqslant\langle\varnothing\rangle-\vec{F}$. For a given stem $s$ and sequence $\vec{F}$ as above, we define $s \wedge \vec{F}=p \wedge \vec{F}$, where $p$ is the weakest condition with stem $s$.

It is easy to see that $\mathbb{P}(\mu, \vec{\gamma}, \vec{\delta}, \vec{U}, \vec{K})$ is $\kappa^{+\gamma}$-centered, and a density argument shows that it forces all cardinals in $\left[\kappa, \kappa^{+\gamma}\right]$ to have countable cofinality. The fact that not more damage is done than intended is a consequence of the Prikry Property, which follows from a more basic combinatorial property. If $\mathbb{P}$ is a partial order and $c: \mathbb{P} \rightarrow\{0,1,2\}$, we say $c$ is a decisive coloring if whenever $c(p)>0$ and $q \leqslant p$, then $c(q)=c(p)$. 
Lemma 3.6 Let c be a decisive coloring of $\mathbb{P}(\mu, \vec{\gamma}, \vec{\delta}, \vec{U}, \vec{K})$.

(1) There is a sequence $\vec{F}$ such that for every condition $p$, every two $r, r^{\prime} \preceq p \wedge \vec{F}$ of the same length have the same color.

(2) For every condition $p$, there is $q \leqslant * p$ such that every two $r, r^{\prime} \leqslant q$ of the same length have the same color.

Proof Let $\mathbb{P}=\mathbb{P}(\mu, \gamma, \delta, U, K)$. For (1), we prove the following claim by induction: For all $n<\omega$ and all decisive colorings of the conditions of length $n$, there is $\vec{F}$ such that for all $m \leqslant n$ and every condition $p$ of length $m$, every two $r, r^{\prime} \preceq p \wedge \vec{F}$ of length $n$ have the same color. Suppose $n=0$ and $c$ is such a coloring. For every $s \in \mathrm{Col}(\mu, \kappa)$, choose if possible some $\vec{F}_{S}$ such that $c\left(\langle s\rangle-\vec{F}_{S}\right)>0$. Using the closure of the higher collapses and diagonal intersections, we may select a single sequence $\vec{F}$ such that $\langle s\rangle \wedge \vec{F} \leqslant\langle s\rangle \frown \vec{F}_{s}$ for all $s$. By decisiveness, $\vec{F}$ witnesses the claim for $n=0$.

Suppose the claim is true for $n-1$. Let $c$ be any decisive coloring of the conditions of length $n$. Using the closure of $\operatorname{Col}\left(\kappa^{+\delta_{n}+2}, j_{U_{n}}(\kappa)\right)^{M_{n}}$, the genericity of $K_{n}$, and the decisiveness of $j_{U_{n}}(c)$, we can find a function $f^{*} \in K_{n}$ such that for every stem $s$ of length $n-1$, if there are some $g$ and $\vec{F}$ such that $g \supseteq f^{*}$ and $s \frown\left\langle j_{U_{n}}\left[\kappa^{+\gamma_{n}}\right], g\right\rangle \frown \vec{F}$ has color $>0$, then $s \frown\left\langle j_{U_{n}}\left[\kappa^{+\gamma_{n}}\right], f^{*}\right\rangle \neg \vec{F}$ already has this color. If $F_{n}$ represents $f^{*}$, then for all stems $s$ of length $n-1$, there is $A_{s} \in U_{n}$ and a color $c_{s}<3$ such that for all $x \in A_{s}$, either there is $\vec{F}^{s, x}=\left\langle F_{k}^{s, x}: n+1 \leqslant k<\omega\right\rangle$ such that $s \frown\left\langle x, F_{n}(x)\right\rangle \frown \vec{F}^{s, x}$ has color $c_{s}>0$, or for all $x \in A_{s}$ and all $g \supseteq F_{n}(x)$, any $p$ of length $n$ with stem ${ }_{s} \frown\langle x, g\rangle$ has color 0 . Let $A$ be the diagonal intersection of the sets $A_{s}$. Using the directed-closure of the filters $K_{k}$ and diagonal intersections, we may select a single sequence $\vec{F}$ that plays the role of $\vec{F}^{s, x}$ for all $s$ and $x$. Putting $\vec{F}^{\prime}=\left\langle F_{n}\lceil A\rangle \frown \vec{F}\right.$, we have that for any condition $p$ of length $n-1$, all $q \preceq p \wedge \vec{F}^{\prime}$ of length $n$ have the same color. This defines a decisive coloring $c^{\prime}$ of the conditions of length $n-1$ of the form $p \wedge \vec{F}^{\prime}$, by coloring them whatever color an arbitrary length- $n$ end-extension receives. By induction, there is $\vec{F}^{\prime \prime}$ such that for every $m \leqslant n-1$, for every condition $p$ of length $m$, every $q \preceq p \wedge \vec{F}^{\prime \prime}$ of length $n-1$ receives the same color under $c^{\prime}$. This means that every such $p \wedge \vec{F}^{\prime \prime}$ receives the same color under $c$ when end-extended to a condition of length $n$.

To finish the argument for (1), let $c$ be a decisive coloring of $\mathbb{P}$. We have for each $n$ a sequence $\vec{F}_{n}$ such that the restriction of $c$ to conditions of length $n$ satisfies the inductive claim. Using the countable closure of the filters $K_{m}$, we can find the desired $\vec{F}$ by taking a lower bound to all the conditions of the form $\langle\varnothing\rangle-\vec{F}_{n}$.

For (2), let $\vec{F}$ be given by (1) and let $p \in \mathbb{P}$. If there is $s \leqslant \operatorname{stem}(p)$ such that some end-extension of $s \wedge \vec{F}$ has color $>0$, then pick such an $s$ which achieves such a color $c^{*}$ by end-extending to length $n$, where $n$ is as small as possible. Then every $r, r^{\prime} \leqslant s \frown \vec{F}$ have color 0 if their length is $<n$, and color $c^{*}$ otherwise.

Corollary $3.7\left\langle\mathbb{P}(\mu, \vec{\gamma}, \vec{\delta}, \vec{U}, \vec{K}), \leqslant, \leqslant{ }^{*}\right\rangle$ is a Prikry-type forcing.

Proof If $\sigma$ is a sentence in the forcing language of $\mathbb{P}(\mu, \vec{\gamma}, \vec{\delta}, \vec{U}, \vec{K})$, then we color a condition 0 if it does not decide $\sigma, 1$ if it forces $\sigma$, and 2 if it forces $\neg \sigma$. This is decisive, so for every $p$, there is $q \leqslant{ }^{*} p$ such that all extensions of $q$ of the same length have the same color. If $q$ does not decide $\sigma$, then there are $r, r^{\prime} \leqslant q$ of the same length forcing opposite decisions about $\sigma$, contradicting the property of $q$. 
Case 2 (of Theorem 3.3): $\tau(\alpha)>\tau(\beta)=\gamma$. Again, we have $\iota(\beta) \geqslant \alpha$, so let $\xi=$

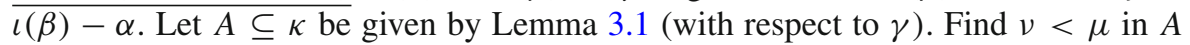
such that $v$ is $v^{+\gamma+1}$-supercompact. Let $G \subseteq \operatorname{Col}\left(v^{+\gamma+\xi+2}, \mu\right)$ be generic over $V$. In $V[G],\left(\mu^{+\gamma+1}, \mu^{+\gamma}\right) \rightarrow v^{+\gamma}\left(v^{+\gamma+1}, v^{+\gamma}\right)$ holds, and $v$ is still $v^{+\gamma+1}$-supercompact. Let $\vec{\gamma}=\left\langle\gamma_{i}: 1 \leqslant i<\omega\right\rangle$ be an increasing sequence converging to $\gamma$. Since $\tau(\alpha)>\gamma$, we may find a nondecreasing sequence $\vec{\alpha}=\left\langle\alpha_{i}: 1 \leqslant i<\omega\right\rangle$ such that $\gamma \leqslant \alpha_{1}$ and $\sum_{i} \alpha_{i}=\alpha$.

Since $v$ is $v^{+\gamma+1}$-supercompact, we can construct $\vec{U}$ and $\vec{K}$ as above according to the sequences $\vec{\gamma}, \vec{\alpha}$. Let $H \subseteq \mathbb{P}(\omega, \vec{\gamma}, \vec{\alpha}, \vec{U}, \vec{K})$ be generic over $V[G]$. Since this forcing is $v^{+\gamma+1}$-c.c., Chang's Conjecture is preserved. In the extension, $v=\aleph_{\alpha}$, $\left(\nu^{+\gamma+1}\right)^{V[G]}=\left(\nu^{+}\right)^{V[G][H]}$, and $\mu^{+\gamma}=\aleph_{\alpha+\xi+\gamma}=\aleph_{\beta}$.

The third case requires a more detailed analysis of the Gitik-Sharon forcing. Suppose $\mathbb{P}(\mu, \vec{\gamma}, \vec{\delta}, \vec{U}, \vec{K})$ is built as above, around a sufficiently supercompact $\kappa$. Associated to a generic filter $G$ are sequences $\left\langle x_{n}: 1 \leqslant n<\omega\right\rangle$, and $\left\langle C_{n}: n<\omega\right\rangle$ determined by the stems of all conditions in $G$, where $C_{0}$ is generic for $\operatorname{Col}\left(\mu, \kappa_{1}\right)$, and for $n \geqslant 1, C_{n}$ is generic for $\operatorname{Col}\left(\kappa_{n}^{+\delta_{n}+2}, \kappa_{n+1}\right)$ and $x_{n} \in \mathcal{P}_{\kappa}\left(\kappa^{+\gamma_{n}}\right)$. From this sequence, we can recover $G$ by taking all conditions $\left\langle f_{0}, x_{1}, f_{1}, \ldots, x_{n}, f_{n}, F_{n+1}, \ldots\right\rangle$ such that:

(1) $\left\langle x_{i}: 1 \leqslant i \leqslant n\right\rangle$ is an initial segment of $\left\langle x_{i}: 1 \leqslant i<\omega\right\rangle$.

(2) For $i \leqslant n, f_{i} \in C_{i}$.

(3) For $i>n, x_{i} \in \operatorname{dom} F_{i}$, and $F_{i}\left(x_{i}\right) \in C_{i}$.

The collection of such conditions is a filter containing $G$, so it must equal $G$ by the maximality of generic filters.

Lemma 3.8 Let $V$ be a model of set theory, and let $\left\langle\mathbb{P}_{i}, \kappa_{i}, G_{i}: i<n\right\rangle$ be such that:

(1) $\left\langle\kappa_{i}: i<n\right\rangle$ is an increasing sequence of regular cardinals in $V$.

(2) For each $i, \mathbb{P}_{i}$ is a partial order in $V$ that is $\left(\kappa_{i}, \kappa_{i}\right)$-distributive and of size $\leqslant \kappa_{i+1}$.

(3) For each $i, G_{i}$ is $\mathbb{P}_{i}$-generic over $V$.

Then $\prod_{i<n} G_{i}$ is $\prod_{i<n} \mathbb{P}_{i}$-generic over $V$.

Proof We show this by induction on $m \leqslant n$. Suppose that $\prod_{i<m} G_{i}$ is $\prod_{i<m} \mathbb{P}_{i^{-}}$ generic over $V$. Since $\mathbb{P}_{m}$ is $\left(\kappa_{m}, \kappa_{m}\right)$-distributive, forcing with it adds no antichains to $\prod_{i<m} \mathbb{P}_{i}$. Thus $\prod_{i<m} G_{i}$ is $\prod_{i<m} \mathbb{P}_{i}$-generic over $V\left[G_{m}\right]$, and so $\prod_{i \leqslant m} G_{i}$ is $\prod_{i \leqslant m} \mathbb{P}_{i}$-generic over $V$.

Lemma $3.9(\vec{x}, \vec{C})$ generates a generic for $\mathbb{P}(\mu, \vec{\gamma}, \vec{\delta}, \vec{U}, \vec{K})$ over $V$ iff the following hold:

(1) For every sequence $\vec{F}=\left\langle F_{n}: 1 \leqslant n<\omega\right\rangle$ such that $\langle\varnothing\rangle \frown \vec{F}$ is a condition of length 0 , there is $m$ such that for all $n \geqslant m, x_{n} \in \operatorname{dom} F_{n}$ and $F_{n}\left(x_{n}\right) \in C_{n}$.

(2) $C_{0}$ is generic for $\operatorname{Col}\left(\mu, \kappa_{1}\right)$, and $C_{n}$ is generic for $\operatorname{Col}\left(\kappa_{n}^{+\delta_{n}+2}, \kappa_{n+1}\right)$ for all $n>0$.

Proof The forward direction is clear. For the reverse direction, let $D \in V$ be a dense open subset of $\mathbb{P}=\mathbb{P}(\mu, \vec{\gamma}, \vec{\delta}, \vec{U}, \vec{K})$, and let $G$ be the filter generated by $(\vec{x}, \vec{C})$. Let $c: \mathbb{P} \rightarrow 2$ be defined by $c(p)=0$ if $p \notin D$ and $c(p)=1$ otherwise. This is decisive, so let $\vec{F}$ be given by Lemma 3.6. Let $m$ be given by (1). 
Consider the condition $p=\left\langle\varnothing, x_{1}, \varnothing, \ldots, x_{m-1}, \varnothing\right\rangle \frown\left\langle F_{i}: m \leqslant i<\omega\right\rangle$. Let $D^{\prime}=$ $\{q \in D: q \leqslant p\}$. $D^{\prime}$ projects to a dense subset of $\operatorname{Col}\left(\mu, \kappa_{1}\right) \times \operatorname{Col}\left(\kappa_{1}^{+\delta_{1}+2}, \kappa_{2}\right) \times$ $\cdots \times \operatorname{Col}\left(\kappa_{m-1}^{+\delta_{m-1}+2}, \kappa_{m}\right)$. By (2) and Lemma 3.8, there is a sequence $\left\langle f_{i}: i<m\right\rangle$ that is in the projection of $D^{\prime}$ intersected with $C_{0} \times \cdots \times C_{m-1}$. Thus there is some condition of the form

$$
\left\langle f_{0}, x_{1}, f_{1}, \ldots, x_{m-1}, f_{m-1}, y_{m}, f_{m}, \ldots, y_{n}, f_{n}, F_{n+1}^{\prime}, \ldots\right\rangle
$$

that is in $D^{\prime}$. But by the homogeneity property of $\vec{F}$, we also have that

$$
\left\langle x_{0}, f_{0}, \ldots, x_{m-1}, f_{m-1}, x_{m}, F_{m}\left(x_{m}\right), \ldots, x_{n}, F_{n}\left(x_{n}\right), F_{n+1}, \ldots\right\rangle \in D
$$

Therefore, $D \cap G \neq \varnothing$.

Case 3 (of Theorem 3.3): $0<\tau(\alpha)=\gamma<\tau(\beta)$. Let $\delta=\beta-\iota(\alpha)$. We can find a nondecreasing sequence $\vec{\delta}=\left\langle\delta_{i}: 1 \leqslant i<\omega\right\rangle$ such that $\delta_{1} \geqslant \gamma$ and $\sum_{i} \delta_{i}=\delta$. Let $\vec{\gamma}=\left\langle\gamma_{i}: 1 \leqslant i<\omega\right\rangle$ be an increasing sequence converging to $\gamma$. Let $j$ be an embedding witnessing that $\kappa$ is $\kappa^{+\gamma+1}$-supercompact, and let $A \subseteq \kappa$ be given by Lemma 3.1 (with respect to $\gamma$ ). For each $n \geqslant 1$, let $U_{n}$ be a $\kappa$-complete normal measure on $\mathcal{P}_{\kappa}\left(\kappa^{+\gamma_{n}}\right)$ derived from $j$, so that $A$ is in the projection of each $U_{n}$ to $\kappa$. Let $\mu=\aleph_{\iota(\alpha)+1}$, and let us force with $\mathbb{P}=\mathbb{P}(\mu, \vec{\gamma}, \vec{\delta}, \vec{U}, \vec{K})$ for where $\vec{K}$ is a sequence of filters as in the construction.

Let $p_{0}$ be a condition of length 0 forcing every Prikry point to be in $A$. Let $p_{1} \leqslant p_{0}$ be a condition of length 1 deciding the statement $\sigma:="\left(\kappa^{+}, \kappa\right) \rightarrow\left(\mu^{+\gamma+1}, \mu^{+\gamma}\right)$." We claim $p_{1} \Vdash \sigma$.

Let us define an iteration of ultrapowers. Let $N_{1}=V$. Given a commuting system of elementary embeddings $j_{m, m^{\prime}}: N_{m} \rightarrow N_{m^{\prime}}$ for $1 \leqslant m \leqslant m^{\prime} \leqslant n$, let $j_{n, n+1}: N_{n} \rightarrow \operatorname{Ult}\left(N_{n}, j_{1, n}\left(U_{n+1}\right)\right)=N_{n+1}$ be the ultrapower embedding, and let $j_{m, n+1}=j_{n, n+1} \circ j_{m, n}$ for $1 \leqslant m<n$. For $1 \leqslant n<\omega$, let $j_{n, \omega}: N_{n} \rightarrow N_{\omega}$ be the direct limit embedding. $N_{\omega}$ is well-founded, and thus can be identified with a transitive class, because of the following generalization of a well-known theorem of Gaifman (see [25]).

Fact 3.10 If $\mathcal{E}$ is a set of countably complete ultrafilters, and $j_{\alpha, \beta}: N_{\alpha} \rightarrow N_{\beta}, \alpha<$ $\beta \leqslant \theta$, is a system of elementary embeddings defined by taking at each $\alpha<\theta$ the ultrapower map $j_{\alpha, \alpha+1}: N_{\alpha} \rightarrow \operatorname{Ult}\left(N_{\alpha}, U\right)=N_{\alpha+1}$ for some $U \in j_{0, \alpha}(\mathcal{E})$, and taking direct limits at limit stages, then each $N_{\alpha}$ is well-founded.

Let stem $\left(p_{1}\right)=\left\langle f_{0}, x_{1}, f_{1}\right\rangle$ and $C_{0} \times C_{1} \subseteq \operatorname{Col}\left(\mu, \kappa_{1}\right) \times \operatorname{Col}\left(\kappa_{1}^{+\delta_{1}+2}, \kappa\right)$ be a filter that contains $\left\langle f_{0}, f_{1}\right\rangle$ and is generic over $V$. For $n>1$, let $y_{n}=j_{n-1, n}\left[j_{1, n-1}\left(\kappa^{+\gamma_{n}}\right)\right]$, and let $x_{n}=j_{n, \omega}\left(y_{n}\right)$, and let $C_{n}=j_{1, n-1}\left(K_{n}\right)$.

Claim $3.11\left\langle x_{n}: 1 \leqslant n<\omega\right\rangle$ and $\left\langle C_{n}: n<\omega\right\rangle$ together generate a generic filter for $j_{1, \omega}(\mathbb{P})$ over $N_{\omega}$.

Proof We need to verify the two conditions of Lemma 3.9. For (1), suppose $\vec{F}=$ $\left\langle F_{n}: 1 \leqslant n<\omega\right\rangle$ is such that $\left\langle\varnothing \supset \vec{F} \in j_{1, \omega}(\mathbb{P})\right.$ is a condition of length 0 . Let $m<\omega$ 
be such that $\vec{F}=j_{m, \omega}\left(\vec{F}^{\prime}\right)$ for some $\vec{F}^{\prime}$. For $n \geqslant m$, we have dom $j_{m, n}\left(F_{n+1}^{\prime}\right) \in$ $j_{1, n}\left(U_{n+1}\right)$, and $N_{n} \models\left[j_{m, n}\left(F_{n+1}^{\prime}\right)\right]_{j_{1, n}\left(U_{n+1}\right)} \in j_{1, n}\left(K_{n+1}\right)$. Thus for $n \geqslant m, y_{n+1} \in$ $\operatorname{dom} j_{m, n+1}\left(F_{n+1}^{\prime}\right)$ and $f_{n+1}:=j_{m, n+1}\left(F_{n+1}^{\prime}\right)\left(y_{n+1}\right) \in C_{n+1}$. Note that $f_{n+1}$ is an object of $\operatorname{rank}<j_{1, n+1}(\kappa)=\operatorname{crit}\left(j_{n+1, \omega}\right)$. Thus for $n>m, x_{n} \in \operatorname{dom} F_{n}$ and $f_{n}=F_{n}\left(x_{n}\right) \in C_{n}$.

To verify (2), note that for each $n>1, N_{n-1} \models j_{1, n-1}\left(K_{n}\right)$ is generic for $\operatorname{Col}\left(j_{1, n-1}\left(\kappa^{+\delta_{n}+2}\right), j_{1, n}(\kappa)\right)$ over $N_{n}$. It is also generic over the submodel $N_{\omega}$. Note also for each $n>1, \kappa_{n}:=x_{n} \cap j_{1, \omega}(\kappa)=j_{1, n-1}(\kappa)$.

Let $G$ be the generated filter for $j_{1, \omega}(\mathbb{P})$. Note that $j_{1, \omega}\left(p_{1}\right) \in G$. We claim that $N_{\omega}[G]$ is closed under $\kappa$-sequences from $V\left[C_{0} \times C_{1}\right]$. Since $C_{0} \times C_{1}$ is generic for a forcing of size $\kappa$, it suffices to show that $N_{\omega}\left[\left\langle x_{n}: 2 \leqslant n<\omega\right\rangle\right]$ is closed under $\kappa$ sequences from $V$, an idea due to Bukovsky [3] and independently to Dehornoy [5]. This follows from the fact that every element of $N_{\omega}$ is of the form $j_{1, \omega}(f)\left(x_{2}, \ldots, x_{n}\right)$ for some $f \in V$ and some $n<\omega$. Let $\left\langle f_{\alpha}: \alpha<\kappa\right\rangle$ be a sequence of functions in $V$, such that for each $\alpha$, there is $n_{\alpha}$ such that dom $f_{\alpha}=\mathcal{P}_{\kappa}\left(\kappa+\gamma_{2}\right) \times \cdots \times \mathcal{P}_{\kappa}\left(\kappa^{+\gamma_{n_{\alpha}}}\right)$. Then $\left\langle j_{1, \omega}\left(f_{\alpha}\right)\left(x_{2}, \ldots, x_{n_{\alpha}}\right): \alpha<\kappa\right\rangle$ can be computed from $j_{1, \omega}\left(\left\langle f_{\alpha}: \alpha<\kappa\right\rangle\right)$ and $\left\langle x_{n}: 2 \leqslant n<\omega\right\rangle$.

For all $\alpha<j_{1, \omega}(\kappa)$, there are $n<\omega$ and $\beta<j_{1, n}(\kappa)$ such that $\alpha=j_{n, \omega}(\beta)$, and $\alpha=\beta$ since $\operatorname{crit}\left(j_{n, \omega}\right)=j_{1, n}(\kappa)$. By GCH and the nature of the measures, for $2 \leqslant n<\omega, \kappa^{+\gamma_{n}}<j_{1, n}(\kappa)<\kappa^{+\gamma}$. Therefore, $j_{1, \omega}(\kappa)=\kappa^{+\gamma}$. Furthermore, an easy counting argument shows that $j_{1, \omega}\left(\kappa^{+\gamma+1}\right)=\kappa^{+\gamma+1}$.

By Lemma 3.1, $V\left[C_{0} \times C_{1}\right]=\left(\kappa^{+\gamma+1}, \kappa^{+\gamma}\right) \rightarrow\left(\mu^{+\gamma+1}, \mu^{+\gamma}\right)$. Let $\mathfrak{A} \in N_{\omega}[G]$ be an algebra on $\kappa^{+\gamma+1}=\left(j_{1, \omega}(\kappa)^{+}\right)^{N_{\omega}[G]}$. In $V\left[C_{0} \times C_{1}\right]$, there is $\mathfrak{B} \prec \mathfrak{A}$ of size $\mu^{+\gamma+1}$ such that $\left|\mathfrak{B} \cap \kappa^{+\gamma}\right|=\mu^{+\gamma}$. By the closure of $N_{\omega}[G], \mathfrak{B} \in N_{\omega}[G]$. This shows that $N_{\omega}[G]$ satisfies the desired instance of Chang's Conjecture, and thus by elementarity that $p_{1}$ forces $\left(\kappa^{+}, \kappa\right) \rightarrow\left(\mu^{+\gamma+1}, \mu^{+\gamma}\right)$. This completes the proof of Theorem 3.3.

Corollary 3.12 Suppose $\mathbb{P}=\mathbb{P}(\mu, \gamma, \delta, U, K)$ is as above. Then there is a condition $p \in \mathbb{P}$ of length 0 that forces

$$
\begin{aligned}
\left(\mu^{+\delta+1}, \mu^{+\delta}\right) & \rightarrow\left(\mu^{+\sum_{1}^{n} \delta_{i}+\gamma+1}, \mu^{+\sum_{1}^{n} \delta_{i}+\gamma}\right) \\
& \rightarrow\left(\mu^{+\sum_{1}^{m} \delta_{i}+\gamma+1}, \mu^{+\sum_{1}^{m} \delta_{i}+\gamma}\right) \rightarrow\left(\mu^{+\gamma+1}, \mu^{+\gamma}\right)
\end{aligned}
$$

for $1 \leqslant m<n<\omega$.

Proof Note that it is forced that $\mu^{+\gamma}=\kappa_{1}^{+\gamma}$, and for each $n \geqslant 1, \kappa_{n}^{+\delta_{n}+\gamma}=\kappa_{n+1}^{+\gamma}=$ $\mu^{+} \sum_{1}^{n} \delta_{i}+\gamma$. Let $p$ be a condition of length 0 that forces all Prikry points to be in the set $A$ given by Lemma 3.1. Fix $1 \leqslant m<n<\omega$, and let $q \leqslant p$ be a condition of length $n$. By Lemma 3.5, $\mathbb{P} \uparrow q$ is isomorphic to a restriction of

$$
\operatorname{Col}\left(\mu, \kappa_{1}\right) \times \cdots \times \operatorname{Col}\left(\kappa_{n-1}^{+\delta_{n-1}+2}, \kappa_{n}\right) \times \mathbb{P}\left(\kappa_{n}^{+\delta_{n}+2}, \gamma^{\prime}, \delta^{\prime}, U^{\prime}, K^{\prime}\right)
$$

where $s^{\prime}$ denotes the shift of a sequence $s$ by $n$. By Lemma 3.1, this product forces $\left(\kappa_{n}^{+\gamma+1}, \kappa_{n}^{+\gamma}\right) \rightarrow\left(\kappa_{m}^{+\gamma+1}, \kappa_{m}^{+\gamma}\right) \rightarrow\left(\mu^{+\gamma+1}, \mu^{+\gamma}\right)$. The last two terms of the product 
are isomorphic to a restriction of $\mathbb{P}\left(\kappa_{n-1}^{+\delta_{n-1}+2}, \vec{\gamma}^{\prime \prime}, \vec{\delta}^{\prime \prime}, \vec{U}^{\prime \prime}, \vec{K}^{\prime \prime}\right)$ to a condition of length 1 , where $s^{\prime \prime}$ denotes the shift of the original sequence $s$ by $n-1$. By the argument for Case 3 of Theorem 3.3, this forces $\left(\kappa_{n}^{+\sum_{n}^{\infty} \delta_{i}+1}, \kappa_{n}^{+\sum_{n}^{\infty} \delta_{i}}\right) \rightarrow\left(\kappa_{n}^{+\gamma+1}, \kappa_{n}^{+\gamma}\right)$.

Our methods are not limited to getting $\left(\aleph_{\beta+1}, \aleph_{\beta}\right) \rightarrow\left(\aleph_{\alpha+1}, \aleph_{\alpha}\right)$ where $\alpha$ and $\beta$ are countable. For example, if we opt not to interleave collapses in the Gitik-Sharon forcing, we obtain:

Porism 3.13 Let $\alpha \geqslant \omega$ be a countable limit ordinal, and let $\kappa$ be a $\kappa^{+\alpha+1}$-supercompact cardinal. Then there is a generic extension in which $\left(\lambda^{+}, \lambda\right) \rightarrow\left(\aleph_{\alpha+1}, \aleph_{\alpha}\right)$, and another in which $\left(\lambda^{+\alpha+1}, \lambda^{+\alpha}\right) \rightarrow\left(\lambda^{+}, \lambda\right)$, where in both cases $\operatorname{cf}(\lambda)=\omega$ and $\aleph_{\lambda}=\lambda$.

\section{Singular global Chang's Conjecture below $\aleph_{\omega^{\omega}}$}

In this section we will prove the following theorem:

Theorem 4.1 If there is a model of ZFC with a cardinal $\delta$ which is $\delta^{+\omega+1}$. supercompact and Woodin for supercompactness, then there is a model in which $\left(\aleph_{\alpha+1}, \aleph_{\alpha}\right) \rightarrow\left(\aleph_{\beta+1}, \aleph_{\beta}\right)$ holds for all limit $\beta<\alpha<\omega^{\omega}$ (including $\beta=0$ ).

This theorem is an attempt to strengthen Corollary 3.2, into a global result. Unfortunately, we do not know how to obtain the desired global result, or even the more natural one in which Chang's Conjecture holds between $\left(\aleph_{\alpha+1}, \aleph_{\alpha}\right)$ and $\left(\aleph_{\beta+1}, \aleph_{\beta}\right)$ for all $\beta<\alpha$ countable limit ordinals. We believe that this is a limitation of our method and not an actual ZFC-barrier.

Before diving into the technical details, let us sketch the main ideas behind the forcing construction: After a suitable preparation, we obtain a model in which many instances of Chang's Conjecture occur between pairs of cardinals of the form $\kappa^{+\omega}$ and its successor and $\mu^{+\omega}$ and its successor. In this model we also have many supercompact cardinals, and this is the reason that we start with a stronger large cardinal hypothesis.

In order to obtain more instances of Chang's Conjecture, we need to apply the "tail changing" forcing, which is a Prikry-type forcing resembling the Gitik-Sharon forcing [12]. Since we would like to do that simultaneously for more than a single pair of cardinals, we define a Magidor- or Radin-like variant of the Gitik-Sharon forcing. Unfortunately, the diagonal nature of the forcing does not allow us to use a Mitchellincreasing sequence of measures, and we are forced to let the domain of measures increase (a similar issue was encountered in [2]). This limits the result of the theorem.

Definition 4.2 A cardinal $\delta$ is called Woodin for supercompactness when for every $A \subseteq \delta$, there is $\kappa<\delta$ such that for all $\lambda \in[\kappa, \delta)$, there is a normal $\kappa$-complete ultrafilter $U$ on $\mathcal{P}_{\kappa}(\lambda)$ such that $j_{U}(A) \cap \lambda=A \cap \lambda$.

Like Woodin cardinals, Woodin for supercompactness cardinals need not be even weakly compact, but they have higher consistency strength than supercompact cardinals. Every almost-huge cardinal is Woodin for supercompactness. Woodin for supercompact cardinals are the same as Vopěnka cardinals (see [19]). 
Lemma 4.3 Suppose GCH and $\delta$ is $\delta^{+\omega+1}$-supercompact and Woodin for supercompactness. Then there is a model of $\mathrm{ZFC}$ in which $\mathrm{GCH}$ holds, there is a supercompact cardinal, and for all $\alpha<\beta$,

$$
\left(\beta^{+\omega+1}, \beta^{+\omega}\right) \rightarrow\left(\alpha^{+\omega+1}, \alpha^{+\omega}\right) .
$$

Furthermore, any such instance of Chang's Conjecture is preserved by forcing over this model with a $\left(\alpha^{+\omega+1}, \alpha^{+\omega+1}\right)$-distributive forcing of size $<\beta^{+\omega}$.

Proof Let $A \subseteq \delta$ be given by Lemma 3.1. Let $\left\langle\alpha_{i}: i<\delta\right\rangle$ enumerate the closure of $A$. Force with the following Easton support iteration $\left\langle\mathbb{P}_{i}, \dot{\mathbb{Q}}_{j}: i \leqslant \delta, j<\delta\right\rangle$ :

(1) $\mathbb{Q}_{0}=\operatorname{Col}\left(\omega, \alpha_{0}^{+\omega}\right) * \operatorname{Col}\left(\alpha_{0}^{+\omega+2}, \alpha_{1}\right)$.

(2) If $i>0$ and $\alpha_{i} \in A$, $\Vdash_{i} \dot{\mathbb{Q}}_{i}=\operatorname{Col}\left(\alpha_{i}^{+\omega+2}, \alpha_{i+1}\right)$.

(3) If $i>0$ and $\alpha_{i} \notin A$, $\Vdash_{i} \dot{\mathbb{Q}}_{i}=\operatorname{Col}\left(\alpha_{i}^{+}, \alpha_{i+1}\right)$.

It is easy to see that this iteration forces that for all infinite $\alpha<\delta$,

$$
\left(\alpha^{+\omega}\right)^{V^{\mathbb{P}_{\delta}}}=\left(\beta^{+\omega}\right)^{V}
$$

for some $\beta \in A$. By standard arguments, $\delta$ remains inaccessible in $V^{\mathbb{P}_{\delta}}$.

Suppose that in $V^{\mathbb{P}_{\delta}}, \alpha<\alpha^{+\omega}<\beta<\delta$, and let $i<j$ be such that

$$
\left(\alpha^{+\omega}\right)^{V^{\mathbb{P}_{\delta}}}=\left(\alpha_{i}^{+\omega}\right)^{V} \quad \text { and } \quad\left(\beta^{+\omega}\right)^{V^{\mathbb{P}_{\delta}}}=\left(\alpha_{j}^{+\omega}\right)^{V}
$$

Then $\mathbb{P}_{\delta}$ factors as $\mathbb{P}_{i} * \mathbb{P}_{j} / \mathbb{P}_{i} * \mathbb{P}_{\delta} / \mathbb{P}_{j}$, where $\left|\mathbb{P}_{i}\right| \leqslant \alpha_{i}^{+\omega}, \mathbb{P}_{j} / \mathbb{P}_{i}$ is forced to be $\alpha_{i}^{+\omega+2}$ closed and of size $\leqslant \alpha_{j}$, and $\mathbb{P}_{\delta} / \mathbb{P}_{j}$ is forced to be $\alpha_{j}^{+\omega+2}$-closed.

Suppose $\mathbb{Q}$ is an $\left(\alpha_{i}^{\omega+1}, \alpha_{i}^{\omega+1}\right)$-distributive forcing of size $<\alpha_{j}^{+\omega}$ in $V^{\mathbb{P}_{\delta}}$. Then $\mathbb{Q} \in V^{\mathbb{P}_{j}}$. Since $\mathbb{P}_{i}$ forces that $\mathbb{P}_{j} / \mathbb{P}_{i} * \mathbb{Q}$ is $\left(\alpha_{i}^{+\omega+1}, \alpha_{i}^{+\omega+1}\right)$-distributive, Lemma 3.1 implies that $\mathbb{P}_{j} * \mathbb{Q}$ forces $\left(\alpha_{j}^{+\omega+1}, \alpha_{j}^{+\omega}\right) \rightarrow\left(\alpha_{i}^{+\omega+1}, \alpha_{i}^{+\omega}\right)$. This is preserved by $\mathbb{P}_{\delta} / \mathbb{P}_{j}$, which remains $\left(\alpha_{j}^{+\omega+1}, \infty\right)$-distributive after forcing with $\mathbb{Q}$.

Finally, we need to find a supercompact. In $V$, let $\kappa<\delta$ be given by Woodin for supercompactness with respect to $A$. Let $\lambda>\kappa$ be an inaccessible limit point of $A$. Let $U$ be a normal $\kappa$-complete ultrafilter on $\mathcal{P}_{\kappa}(\lambda)$ such that $j_{U}(A) \cap \lambda=A \cap \lambda$. We have that $j_{U}\left(\mathbb{P}_{\kappa}\right)=\mathbb{P}_{\kappa} * \mathbb{P}_{\lambda} / \mathbb{P}_{\kappa} * \mathbb{Q}$, for some $\mathbb{Q}$ that is forced to be $\lambda^{+}$-closed. Let $G_{\delta} \subseteq \mathbb{P}_{\delta}$ be generic, and let $G_{\lambda}=G_{\delta}\left\lceil\mathbb{P}_{\lambda}\right.$. By GCH, $j_{U}(\kappa)<\lambda^{++}$and $j_{U}\left(\lambda^{++}\right)=\lambda^{++}$, so we may build $H \subseteq \mathbb{Q}$ in $V\left[G_{\lambda}\right]$ that is generic over $M\left[G_{\lambda}\right]$. Thus we can extend the embedding to $j: V\left[G_{\kappa}\right] \rightarrow M\left[G_{\lambda} * H\right]$. Since $M\left[G_{\lambda} * H\right]$ is $\lambda$-closed in $V\left[G_{\lambda}\right]$ and $\mathbb{P}_{\lambda} / G_{\kappa}$ is $\kappa$-directed-closed, there is $p \in j_{U}\left(\mathbb{P}_{\lambda}\right) /\left(G_{\lambda} * H\right)$ below $j\left[G_{\lambda} / G_{\kappa}\right]$. Since $\left|\mathbb{P}_{\lambda}\right|=\lambda$ and $j_{U}\left(\lambda^{+}\right)<\lambda^{++}$, we can build $K \subseteq j_{U}\left(\mathbb{P}_{\lambda}\right) /\left(G_{\lambda} * H\right)$ below $p$ in $V\left[G_{\lambda}\right]$ that is generic over $M\left[G_{\lambda} * H\right]$. Thus we can extend the embedding to $j: V\left[G_{\lambda}\right] \rightarrow M\left[G_{\lambda} * H * K\right]$. This shows that $\kappa$ is $\lambda$-supercompact in $V\left[G_{\lambda}\right]$, a property that is preserved by $\mathbb{P}_{\delta} / G_{\lambda}$. Thus, $V_{\delta}\left[G_{\delta}\right] \models$ "There is a supercompact cardinal." 
Let us work in a model satisfying the conclusion of the above lemma. We define by induction on $1 \leqslant n \leqslant \omega$ the class of "order- $n$ Gitik-Sharon forcings" (abbreviated by $\mathrm{GS}_{n}$ ). Formally, we fix a large enough regular $\theta$ and define these inductively as subsets of $H_{\theta}$, but it will be clear that choice of $\theta$ is irrelevant, and for $\theta<\theta^{\prime}$, $\mathrm{GS}_{n}^{H_{\theta}}=\mathrm{GS}_{n}^{H_{\theta^{\prime}}} \cap H_{\theta}$. Each order- $n$ forcing will add a club of ordertype $\omega^{n}$ to a large cardinal $\kappa$, consisting of former inaccessibles, while preserving $\kappa$ as a cardinal, collapsing $\kappa^{+\omega \cdot n}$ to $\kappa$, and preserving larger cardinals.

$\mathrm{GS}_{1}$ is the collection of forcings of the form $\mathbb{P}\left(\mu, \vec{\omega}, \overrightarrow{\omega^{2}}, \vec{U}, \vec{K}\right)$, as defined in the previous section, where $\vec{\omega}$ is the identity sequence $\langle 1,2,3, \ldots\rangle$, and $\vec{\omega}^{2}$ is the constant sequence $\langle\omega, \omega, \omega, \ldots\rangle$.

Definition 4.4 A sequence $d=\left\langle U_{\alpha}, K_{\alpha}: \alpha<\omega \cdot n\right\rangle$ is a $\mathrm{GS}_{n}$-sequence if

(1) There is a $\kappa>\omega$ such that each $U_{\alpha}$ is a $\kappa$-complete ultrafilter. We call $\kappa$ the critical point of the sequence $d$.

(2) For $1 \leqslant n<\omega, U_{n}$ is a normal ultrafilter on $\mathcal{P}_{\kappa}\left(\kappa^{+n}\right)$ and for $\omega \leqslant \alpha<\omega \cdot n$ successor, $U_{\alpha}$ is a normal ultrafilter on $\mathcal{P}_{\kappa}\left(H_{\kappa+\alpha}\right)$.

(3) For successor $\alpha<\omega \cdot n$, if $j_{\alpha}: V \rightarrow M_{\alpha}$ is the ultrapower embedding from $U_{\alpha}$, then $K_{\alpha}$ is $\operatorname{Col}\left(\kappa^{+\alpha+\omega+2}, j_{\alpha}(\kappa)\right)^{M_{\alpha}}$-generic over $M_{\alpha}$.

A partial order $\mathbb{P} \in \mathrm{GS}_{n}$ will be determined by the choice of a $\mathrm{GS}_{n}$-sequence $d$ and a regular cardinal $\mu<\operatorname{crit}(d)$. Suppose $n>1$ and that we have defined $\mathrm{GS}_{m}$ for $m<n$, and we have a function defined on pairs $(\mu, d) \in H_{\theta}$ that outputs a partial order $\mathbb{P}(\mu, d) \in \mathrm{GS}_{m}$ whenever $d$ is a sequence of length $\omega \cdot m$ as above and $\mu<\operatorname{crit}(d)$ is regular.

Let $d=\left\langle U_{\alpha}, K_{\alpha}: \alpha<\omega \cdot n\right\rangle$ be as above and let $\mu<\operatorname{crit}(d)$ be regular. Conditions in $\mathbb{P}(\mu, d) \in \mathrm{GS}_{n}$ take the form

$$
p=\left\langle f_{0}, e_{1},\left(x_{1}, a_{1}\right), f_{1}, e_{2},\left(x_{2}, a_{2}\right), f_{2}, \ldots, e_{l},\left(x_{l}, a_{l}\right), f_{l}, \vec{F}\right\rangle .
$$

The stem of $p$ is the initial segment obtained by removing $\vec{F}$. The length of $p$ as above is $l$. We require:

(1) For $1 \leqslant i \leqslant l$ :

(a) $\left|x_{i}\right|<\kappa, x_{i} \prec H_{\kappa+\omega \cdot(n-1)+i}, \kappa_{i}:=x_{i} \cap \kappa$ is inaccessible, the transitive collapse of $x_{i}$ is $H_{\kappa_{i}^{+\omega \cdot(n-1)+i}}$, and $\left\langle U_{\alpha}, K_{\alpha}: \alpha<\omega \cdot(n-1)\right\rangle \in x_{i}$.

(b) Let $\pi: x_{i} \rightarrow H$ be the transitive collapse map. Put $\pi\left(\left\langle U_{\alpha}, K_{\alpha}: \alpha<\omega\right.\right.$ $\cdot(n-1)\rangle):=\left\langle u_{\alpha}^{i}, k_{\alpha}^{i}: \alpha<\omega \cdot(n-1)\right\rangle:=d_{i}$. We require that $d_{i}$ is a $\mathrm{GS}_{n-1}$-sequence, $a_{i}$ is a sequence of functions $\left\langle b_{\alpha}^{i}: \alpha<\omega \cdot(n-1)\right\rangle$ such that $\operatorname{dom}\left(b_{\alpha}^{i}\right) \in u_{\alpha}^{i}$ and $\left[b_{\alpha}^{i}\right]_{u_{\alpha}^{i}} \in k_{\alpha}^{i}$.

(2) $f_{0} \in \operatorname{Col}(\mu, \kappa)$, and if $l>0$, then $\left\langle f_{0} \curlyvee e_{1} \frown a_{1} \in \mathbb{P}\left(\mu, d_{1}\right)\right.$, where $f_{0} \frown e_{1}$ is the stem of the condition.

(3) For $1 \leqslant i<l, x_{i} \in x_{i+1}$, and $\left\langle f_{i}\right\rangle e_{i+1} \frown a_{i+1} \in \mathbb{P}\left(\kappa_{i}^{+\omega \cdot n+2}, d_{i+1}\right)$, where $f_{i} \frown e_{i+1}$ is the stem.

(4) $f_{l} \in \operatorname{Col}\left(\kappa_{l}^{+\omega \cdot n+2}, \kappa\right)$.

(5) $\vec{F}$ is a sequence of functions $\left\langle F_{\alpha}: \alpha<\omega \cdot n\right\rangle$ such that for each $\alpha$, dom $F_{\alpha} \in U_{\alpha}$ and $\left[F_{\alpha}\right]_{U_{\alpha}} \in K_{\alpha}$. 
Suppose we have two conditions

$$
\begin{aligned}
& p=\left\langle f_{0}, e_{1},\left(x_{1}, a_{1}\right), f_{1}, \ldots, e_{l},\left(x_{l}, a_{l}\right), f_{l}, \vec{F}\right\rangle \\
& q=\left\langle f_{0}^{\prime}, e_{1}^{\prime},\left(x_{1}^{\prime}, a_{1}^{\prime}\right), f_{1}^{\prime}, \ldots, e_{m}^{\prime},\left(x_{m}^{\prime}, a_{m}^{\prime}\right), f_{m}^{\prime}, \vec{F}^{\prime}\right\rangle .
\end{aligned}
$$

We put $q \leqslant p$ when:

(1) $m \geqslant l$, and for $1 \leqslant i \leqslant l, x_{i}=x_{i}^{\prime}$.

(2) For $i \leqslant l, f_{i}^{\prime} \supseteq f_{i}$.

(3) For $1 \leqslant i \leqslant l,\left\langle f_{i-1}^{\prime}\right\rangle e_{i}^{\prime} a_{i}^{\prime} \leqslant\left\langle f_{i-1}\right\rangle e_{i} \frown a_{i}$ in the relevant partial order from $\mathrm{GS}_{n-1}$.

(4) For $l<i \leqslant m, x_{i}^{\prime} \in \operatorname{dom} F_{\omega \cdot(n-1)+i}$ and $f_{i}^{\prime} \supseteq F_{\omega \cdot(n-1)+i}\left(x_{i}\right)$.

(5) $\vec{F}\left\lceil\omega \cdot(n-1) \in x\right.$ if $x=x_{i}^{\prime}$ for $l<i \leqslant m$, or if $x \in \operatorname{dom} F_{\omega \cdot(n-1)+k}^{\prime}$ for $k>m$.

(6) Put $f_{k}=F_{\omega \cdot(n-1)+k}\left(x_{k}^{\prime}\right)$ for $l<k<m$. If $l<i \leqslant m$ and $\pi: x_{i} \rightarrow H$ is the transitive collapse map, then $\left\langle f_{i-1}^{\prime} \curlyvee e_{i}^{\prime} a_{i}^{\prime} \leqslant\left\langle f_{i-1} \curlyvee \pi(\vec{F}\lceil\omega \cdot(n-1))\right.\right.$.

(7) For each $\alpha<\omega \cdot n, \operatorname{dom} F_{\alpha}^{\prime} \subseteq \operatorname{dom} F_{\alpha}$, and for each $x \in \operatorname{dom} F_{\alpha}^{\prime}, F_{\alpha}^{\prime}(x) \supseteq F_{\alpha}(x)$.

Finally, we may define the order- $\omega$ forcings which generically stack the order- $n$ forcings for finite $n$. Everything looks quite similar, except now our sequences of functions $\vec{F}$ have length $\omega^{2}$, and stems of length $n>0$ look like stems of length- 1 conditions from forcings in $\mathrm{GS}_{n+1}$.

Remark 4.5 Unlike the standard supercompact Radin forcing (such as in [14]), the generic Radin point $x_{\alpha}$ for limit $\alpha$ is strictly larger than $\bigcup_{\beta<\alpha} x_{\beta}$. This discontinuity plays an important role in the proof of the Prikry Property.

We define some notions to describe the conditions in our forcings. A type-1 sequence is a natural number. For $n>1$, a type- $n$ sequence is a finite sequence of type- $(n-1)$ sequences. We can define inductively a partial order on these sequences. For a type-1 sequence, this is just the usual linear order. If $s=\left\langle t_{1}, \ldots, t_{l}\right\rangle$ and $s^{\prime}=\left\langle t_{1}^{\prime}, \ldots, t_{m}^{\prime}\right\rangle$ are of type- $n$, then we say $s^{\prime} \geqslant s$ when $m \geqslant l$ and $t_{i}^{\prime} \geqslant t_{i}$ for $1 \leqslant i \leqslant m$. It is easy to see by induction that this ordering is upward-directed.

If $p \in \mathbb{P} \in \mathrm{GS}_{1}$, then by the shape of $p$ we mean its length. If $s=\left\langle t_{1}, \ldots, t_{l}\right\rangle$ is a type- $n$ sequence, and

$$
p=\left\langle f_{0}, e_{1},\left(x_{1}, a_{1}\right), f_{1}, \ldots, e_{l},\left(x_{l}, a_{l}\right), f_{l}, \vec{F}\right\rangle \in \mathbb{P} \in \mathrm{GS}_{n},
$$

then we say, inductively, that the stem of $p$ has shape $s$ if each $\left\langle f_{i-1}\right\rangle \frown e_{i} \frown a_{i}$ has shape $t_{i}$. If $s=\left\langle t_{1}, \ldots, t_{l}\right\rangle$ is such that each $t_{i}$ is a type- $i$ sequence, and $p \in \mathbb{P} \in \mathrm{GS}_{\omega}$ takes the same form as above, then we say $p$ has shape $s$ if each $\left\langle f_{i-1}\right\rangle \frown e_{i} \frown a_{i}$ has shape $t_{i}$. Note that if $q \leqslant p$, then the shape of $q$ is greater or equal to the shape of $p$ in the ordering on sequences. Since the shape of a condition only depends on its stem, we will also speak of the shapes of stems and their subsequences.

Suppose $\mathbb{P} \in \mathrm{GS}_{n}$ for $n \leqslant \omega$. For conditions $p, q \in \mathbb{P}$, we say $p \leqslant{ }^{*} q$ if $p \leqslant q$ and they have the same shape. If $p \leqslant q$ and $\operatorname{stem}(p)$ is an initial segment of $\operatorname{stem}(q)$, then we say $p \preceq q$. We have an operation $p \wedge \vec{F}$ defined similarly as before, in the discussion preceding Lemma 3.6. 
Lemma 4.6 Suppose $\mathbb{P}(\mu, d) \in \mathrm{GS}_{n}, \mu>\omega$, and $c: \mathbb{P} \rightarrow 3$ is a decisive coloring.

(1) There is a sequence $\vec{F}$ such that for every condition $p$, every two $r, r^{\prime} \preceq p \wedge \vec{F}$ of the same shape have the same color.

(2) For every condition $p$, there is $q \leqslant *$ such that every two $r, r^{\prime} \leqslant q$ of the same shape have the same color.

Proof The case $n=1$ was proven in Lemma 3.6. Assume $n>1$ and the lemma holds for $\mathrm{GS}_{m}, m<n$. Let $\mathbb{P}(\mu, d) \in \mathrm{GS}_{n}$, with crit $(d)=\kappa$ and $\omega<\mu<\kappa$. Like before, we prove (1) by showing the following claim by induction: For all $l<\omega$ and all decisive colorings of the conditions of length $l$, there is $\vec{F}_{l}$ such that for all $m \leqslant l$ and every condition $p$ of length $m$, every two $r, r^{\prime} \preceq p \wedge \vec{F}$ of the same shape and of length $l$ have the same color. This suffices, since we can find $\vec{F}$ that is a lower bound to the countably many $\vec{F}_{l}$. Suppose $l=0$ and $c$ is such a coloring. For every $s \in \operatorname{Col}(\mu, \kappa)$, choose if possible some $\vec{F}_{S}$ such that $c\left(\langle s\rangle \frown \vec{F}_{S}\right)>0$. Using the directed closure of the collapses and diagonal intersections, we may select a single sequence $\vec{F}$ such that $\langle s\rangle \wedge \vec{F} \leqslant\langle s\rangle \frown \vec{F}_{s}$ for all $s$.

Suppose the claim is true for $m<l$. Let $c$ be a decisive coloring of the conditions of length $l$. For each stem $s=\left\langle f_{0}^{s}, \ldots,\left(x_{l-1}^{s}, a_{l-1}^{s}\right), f_{l-1}^{s}\right\rangle$ of length $l-1$, and each candidate $(x, a)$ for the last node in a one-step extension containing $s$, we can define a coloring $c_{s, x}$ on conditions of the form $\left.\left\langle f_{l-1}^{s}\right\rangle\right\rangle^{\frown} a \in \mathbb{P}\left(\kappa_{l-1}^{+\omega \cdot n+2}, d_{x}\right)$ as follows. First, as in the proof of Lemma 3.6, we find a sequence $\vec{F}=\left\langle F_{\alpha}: \alpha<\omega \cdot n\right\rangle$ such that for each stem $s$, each $x \in \operatorname{dom} F_{\omega \cdot(n-1)+l}$, and each choice of $e$ and $a$ such that there are $f$ and $\vec{H}$ such that $s \frown\langle e,(x, a), f\rangle \frown \vec{H}$ is a condition below $s \frown \vec{F}$ with color $>0$, then already $s \frown\left\langle e,(x, a), F_{\omega \cdot(n-1)+l}(x)\right\rangle \frown \vec{F}$ has this color. We can then define $c_{s, x}\left(\left\langle f_{l-1}^{s} \curlyvee\right\rangle^{-} a\right)=c\left(s \frown\left\langle e,(x, a), F_{\omega \cdot(n-1)+l}(x)\right\rangle \frown \vec{F}\right)$. By the induction hypothesis on the order of Gitik-Sharon forcing, for each such $s, x$, there is a choice of $a_{s, x}$ such that $c_{s, x}\left(\left\langle f_{l-1}^{s}\right\rangle-e-a_{s, x}\right)$ depends only on the shape of $e$, for conditions below $\left\langle f_{l-1}^{s}\right\rangle a_{s, x}$. For each $x$, we can use diagonal intersections to select a sequence $a_{x}$ such that for all $s,\left\langle f_{l-1}^{s}\right\rangle \wedge a_{x} \leqslant\left\langle f_{l-1}^{s}\right\rangle a_{s, x}$.

In the ultrapower by $U=U_{\omega \cdot(n-1)+l}$, the function $x \mapsto a_{x}$ represents a sequence of functions $\vec{G}$ strengthening $\vec{F}\left\lceil\omega \cdot(n-1)=\pi\left(j_{U}(\vec{F}\lceil\omega \cdot(n-1)))\right.\right.$, where $\pi$ is the transitive collapse of $j_{U}\left[H_{\kappa}+\omega \cdot(n-1)+l\right]$. Let $\vec{F}^{\prime}$ be $\vec{F}$ with the intial segment below $\omega \cdot(n-1)$ replaced by $\vec{G}$. Thus we have for each stem $s$ of length $l-1$, a set $A_{s} \in U_{\omega \cdot(n-1)+l}$ such that for all $x \in A_{s}, a_{x}=\pi_{x}\left(F^{\prime} \uparrow \omega \cdot(n-1)\right)$, and the color of $s \frown\left\langle e,\left(x, a_{x}\right), F_{\omega \cdot(n-1)+l}^{\prime}(x)\right\rangle-\vec{F}^{\prime}$ depends only on the shape of $e$, if $\left.\left\langle f_{l-1}^{s}\right\rangle\right\urcorner e^{\frown} a_{x} \leqslant\left\langle f_{l-1}^{s}\right\rangle \frown a_{x}$. Let $A^{*}$ be the diagonal intersection of the $A_{s}$, and let $\vec{F}^{\prime \prime}$ be $\vec{F}^{\prime}$ restricted to $A^{*}$ at coordinate $\omega \cdot(n-1)+l$.

Now for any condition $p$ of shape $\left\langle t_{1}, \ldots, t_{l-1}\right\rangle$, the color under $c$ of any $q \preceq p \wedge \vec{F}^{\prime \prime}$ of shape $\left\langle t_{1}, \ldots, t_{l-1}, t\right\rangle$ depends only on $t$. So for each type- $(n-1)$ sequence $t$, let $c_{t}$ color the length- $(l-1)$ conditions accordingly. Note that each $c_{t}$ inherits decisiveness from $c$. By the induction hypothesis, for each $t$, there is a sequence $\vec{F}_{t}$ such that for all $m<l-1$ and all $p$ of length $m$, every $q \preceq p \wedge \vec{F}_{t}$ of length $l-1$ has a color under $c_{t}$ depending only on the shape of $q$. If $\vec{F}^{\prime \prime \prime}$ is a lower bound to the countably many sequences $\vec{F}_{t}$, then $\vec{F}^{\prime \prime \prime}$ satisfies the inductive claim for $l$. This concludes the argument for (1). 
To show (2), let us assume inductively that it holds for $\mathrm{GS}_{m}, m<n$. Let $\mathbb{P} \in \mathrm{GS}_{n}$, let $c: \mathbb{P} \rightarrow 3$ be decisive, and let $\vec{F}$ be given by (1). Let $p \in \mathbb{P}$, with stem $(p)=$ $\left\langle f_{0}, \ldots, f_{l-1}, e_{l},\left(x_{l}, a_{l}\right), f_{l}\right\rangle$. For every end-extension $q=\operatorname{stem}(p) \frown s \frown \vec{F}$ of $p \wedge \vec{F}$, the color of $q$ depends only on the shape of $s$. Using the closure of $\operatorname{Col}\left(\kappa_{l}^{+\omega \cdot n+2}, \kappa\right)$, we can find $f_{l}^{\prime} \supseteq f_{l}$ such that for every strengthening $s$ of the initial segment of stem $(p)$ before $f_{l}$, and every type- $n$ sequence $t$, if there is $f \supseteq f_{l}^{\prime}$ such that some $s^{\prime}$ of shape $t$ with $s \frown\langle f\rangle \frown s^{\prime} \vec{F} \leqslant p \wedge \vec{F}$ has color $>0$, then already $s \frown\left\langle f_{l}^{\prime}\right\rangle \frown s^{\prime} \vec{F}$ has this color.

Now for each type- $n$ sequence $t$, and each strengthening $s$ of $\operatorname{stem}(p)$ before $f_{l-1}$, we have a coloring $c_{s, t}$ of the conditions $\langle f\rangle \frown e \frown a \leqslant\left\langle f_{l-1}\right\rangle \frown e_{l}^{\frown} a_{l}$ according to the color under $c$ of $s \frown\left\langle f, e,\left(x_{l}, a\right), f_{l}^{\prime}\right\rangle s^{\prime} \frown \vec{F}$, where $s^{\prime}$ is anything of shape $t$, such that the resulting condition is below $p \wedge \vec{F}$. Using the inductive hypothesis and the weak closure of $\mathbb{P}\left(\kappa_{l-1}^{+\omega \cdot n+2}, d_{l}\right)$, we find $\left\langle f_{l-1}^{\prime} \curlyvee e_{l}^{\prime} a_{l}^{\prime} \leqslant *\left\langle f_{l-1}\right\rangle \curlyvee e_{l}^{\frown} a_{l}\right.$ such that any two extensions of the former of the same shape have the same color under every $c_{s, t}$. As a result, we have that for any $s$ strengthening $\operatorname{stem}(p)$ before $f_{l-1}$, for any two $r, r^{\prime}$ of the same shape below $s \smile\left\langle f_{l-1}^{\prime}, e_{l}^{\prime},\left(x_{l}, a_{l}^{\prime}\right), f_{l}^{\prime}\right\rangle \vec{F}$, for which $s$ is an initial segment of both, $c(r)=c\left(r^{\prime}\right)$. We continue this process in the same fashion down the stem of $p$, in a total of $l$ steps, so that at step $k \leqslant l$, we find $\left\langle f_{l-k-1}^{\prime}\right\rangle e_{l-k}^{\prime} \frown a_{l-k}^{\prime} \leqslant{ }^{*}\left\langle f_{l-k-1}\right\rangle \frown e_{l-k} \widetilde{a_{l-k}}$, such that for every strengthening $s$ of the initial segment of stem $(p)$ before $f_{l-k-1}$, any two conditions $r, r^{\prime}$ of the same shape, with $s$ as an initial segment, and below $s^{\frown}\left\langle f_{l-k-1}^{\prime}, e_{l-k}^{\prime},\left(x_{l-k}, a_{l-k}^{\prime}\right), f_{l-k}^{\prime}, \ldots,\left(x_{l}, a_{l}^{\prime}\right), f_{l}^{\prime}, \vec{F}\right\rangle$, we have $c(r)=c\left(r^{\prime}\right)$. Eventually we reach the desired condition $q \leqslant{ }^{*} p$.

The inductive argument for $\mathrm{GS}_{\omega}$ is entirely similar.

Corollary 4.7 If $\mathbb{P}(\mu, d) \in \mathrm{GS}_{n}, 1 \leqslant n \leqslant \omega<\mu$, then $\langle\mathbb{P}(\mu, d), \leqslant, \leqslant *\rangle$ is a Prikrytype forcing. Furthermore, for a condition $p_{0}$ of the form

$$
\left\langle f_{0}, e_{1},\left(x_{1}, a_{1}\right), f_{1}, \ldots, e_{m},\left(x_{m}, a_{m}\right), f_{m}, \vec{F}\right\rangle,
$$

$\mathbb{P}(\mu, d) \uparrow p_{0}$ is canonically isomorphic to

$$
\mathbb{P}\left(\mu, d_{1}\right)\left\lceil\left\langle f_{0}\right\rangle \frown e_{1} \frown a_{1} \times \cdots \times \mathbb{P}\left(\kappa_{m-1}^{+\omega \cdot n+2}, d_{m}\right) \uparrow\left\langle f_{m-1}\right\rangle \frown e_{m} \frown a_{m} \times \mathbb{Q},\right.
$$

where $\mathbb{Q}$ is a weakly $\kappa_{m}^{+\omega \cdot m+2}$-closed Prikry-type forcing.

Proof Let $\sigma$ be a sentence in the forcing language, and color conditions 0 if they do not decide $\sigma, 1$ if they force $\sigma$, and 2 if they force $\neg \sigma$. Let $p \in \mathbb{P}(\mu, d)$, and let $q \leqslant{ }^{*} p$ be such that any two extensions of $q$ of the same shape have the same color. If $q$ does not decide $\sigma$, then by the fact that the ordering on sequences is upward-directed, we can find $r, r^{\prime} \leqslant q$ of the same shape that force opposite decisions about $\sigma$, a contradiction.

For the second claim, the map is the obvious one, where the elements of $\mathbb{Q}$ are the tail-ends beyond place $m$, of conditions below $p_{0}$. Let us write $\mathbb{P}(\mu, d)\left\lceil p_{0}\right.$ as $\mathbb{R} \times \mathbb{Q}$. From any decisive coloring $c$ of the conditions in $\mathbb{Q}$, we can define a decisive coloring $c^{\prime}$ of $\mathbb{R} \times \mathbb{Q}$ by setting $c^{\prime}(r, q)=c(q)$. Given any $q \in \mathbb{Q}$, we can find $p \leqslant *(1, q)$ such that any two $p^{\prime}, p^{\prime \prime} \leqslant p$ of the same shape have the same color under $c^{\prime}$. This means that any two $q^{\prime}, q^{\prime \prime} \leqslant q$ of the same shape have the same color under $c$. Then we apply the argument of the previous paragraph. 
If $\mathbb{P}(\mu, d) \in \mathrm{GS}_{n}$ for $1<n<\omega$, with crit $(d)=\kappa$, and $G \subseteq \mathbb{P}(\mu, d)$ is generic over $V$, then we have a sequence $\left\langle x_{i}, G_{i}: 1 \leqslant i<\omega\right\rangle$ such that:

(1) Each $x_{i}$ is a typical point in $\mathcal{P}_{\kappa}\left(H_{\kappa+\omega \cdot(n-1)+i}\right)$.

(2) $\left\langle x_{i}: 1 \leqslant i<\omega\right\rangle$ is $\in$ - and $\subseteq$-increasing, with $\bigcup_{i} x_{i}=H_{\kappa}+\omega \cdot n$.

(3) $G_{1}$ is $\mathbb{P}\left(\mu, d_{1}\right)$-generic, and for $i>1, G_{i}$ is $\mathbb{P}\left(\kappa_{i-1}^{+\omega \cdot n+2}, d_{i}\right)$-generic, where $\kappa_{i}$ and $d_{i}$ are as in the definition of $\mathrm{GS}_{n}$.

From $\left\langle x_{i}, G_{i}: 1 \leqslant i<\omega\right\rangle$, we can recover $G$ as the collection of all conditions $\left\langle f_{0}, e_{1},\left(x_{1}, a_{1}\right), f_{1}, \ldots, e_{l},\left(x_{l}, a_{l}\right), f_{l}, \vec{F}\right\rangle$ such that:

(1) $\left\langle x_{1}, \ldots, x_{l}\right\rangle$ is an initial segment of $\left\langle x_{i}: 1 \leqslant i<\omega\right\rangle$.

(2) For $i>l, F\left\lceil\omega \cdot(n-1) \in x_{i} \in \operatorname{dom} F_{\omega \cdot n+i}\right.$.

(3) For $1 \leqslant i \leqslant l,\left\langle f_{i-1}\right\rangle \frown e_{i} a_{i} \in G_{i}$.

(4) Putting $f_{i}=F_{\omega \cdot n+i}\left(x_{i}\right)$ for $i>l,\left\langle f_{i-1}\right\rangle \frown \vec{F}\left\lceil\omega \cdot(n-1) \in G_{i}\right.$.

We need the following characterization of genericity, proof of which is essentially the same as for Lemma 3.9:

Lemma 4.8 Suppose $d=\left\langle U_{\alpha}, K_{\alpha}: \alpha<\omega \cdot n\right\rangle$ and $\mathbb{P}(\mu, d) \in \mathrm{GS}_{n}$, with $\omega<$ $\mu<\operatorname{crit}(d)=\kappa$. Suppose in some outer model $W \supseteq V$, there is a sequence $\left\langle x_{i}, G_{i}: 1 \leqslant i<\omega\right\rangle$ as above.

Then this sequence generates a $V$-generic filter $G$ for $\mathbb{P}(\mu, d)$ iff for every sequence

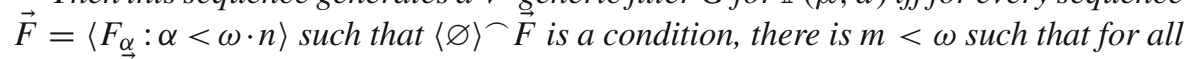
$k \geqslant m, \vec{F}\left\lceil\omega \cdot(n-1) \in x_{k} \in \operatorname{dom} F_{\omega \cdot(n-1)+k}\right.$, and

$$
\left\langle F_{\omega \cdot(n-1)+k}\left(x_{k}\right)\right\rangle \pi_{k+1}\left(\vec{F}\lceil\omega \cdot(n-1)) \in G_{k+1},\right.
$$

where $\pi_{k+1}$ is the transitive collapse of $x_{k+1}$.

To prove the main theorem, we will show by induction that, in a model satisfying the conclusion of Lemma 4.3 , if $\mu=v^{+\omega \cdot k+2}$ and $\mathbb{P}(\mu, d) \in \mathrm{GS}_{n}$, for $1 \leqslant k, n<\omega$, then $\mathbb{P}(\mu, d)$ forces that $\left(v^{+\alpha+1}, v^{+\alpha}\right) \rightarrow\left(v^{+\beta+1}, v^{+\beta}\right)$ holds for all limit ordinals $\omega \leqslant \beta<\alpha \leqslant \omega^{n+1}$. Note that we include the case $v=0$ so that the lower pair may be $\left(\aleph_{1}, \aleph_{0}\right)$.

For the base case, suppose $\mu=v^{+\omega \cdot k+2}$, for $1 \leqslant k<\omega$, and $\mathbb{P}(\mu, d) \in \mathrm{GS}_{1}$, with $\operatorname{crit}(d)=\kappa$. By Lemma 3.5 and the preservation claim of Lemma 4.3, we have that in $V^{\mathbb{P}(\mu, d)},\left(v^{+\omega \cdot i+1}, v^{+\omega \cdot i}\right) \rightarrow\left(v^{+\omega \cdot j+1}, v^{+\omega \cdot j}\right)$ holds for all $1 \leqslant j<i<\omega$. Using again the fact that for $\alpha<\kappa,\left(\kappa^{+\omega+1}, \kappa^{+\omega}\right) \rightarrow\left(\alpha^{+\omega+1}, \alpha^{+\omega}\right)$ is indestructible by any $\alpha^{+\omega+2}$-closed forcing of size $\kappa$, the iterated ultrapower construction in the previous section shows that $\mathbb{P}(\mu, d)$ also forces $\left(\kappa^{+}, \kappa\right) \rightarrow\left(v^{+\omega \cdot i+1}, v^{+\omega \cdot i}\right)$ for $1 \leqslant i<\omega$.

Assuming that the inductive claim holds for $n$, let us first argue for the weaker claim that if $\mu=v^{+\omega \cdot k+2}$, for $1 \leqslant k<\omega$, and $\mathbb{P}(\mu, d) \in \mathrm{GS}_{n+1}$, then $\mathbb{P}(\mu, d)$ forces $\left(v^{+\alpha+1}, v^{+\alpha}\right) \rightarrow\left(v^{+\beta+1}, v^{+\beta}\right)$ to hold for all limit ordinals $\omega \leqslant \beta<\alpha<\omega^{n+2}$ (where the last inequality is strict). A generic $G \subseteq \mathbb{P}(\mu, d)$ introduces a Prikry sequence of generics for $\mathrm{GS}_{n}$ forcings, $\left\langle G_{i}: 1 \leqslant i<\omega\right\rangle$, where $G_{1}$ is generic for $\mathbb{P}\left(\mu, d_{1}\right)$, and for $i \geqslant 2, G_{i}$ is generic for $\mathbb{P}\left(\kappa_{i-1}^{+\omega \cdot(n+1)+2}, d_{i}\right)$. In $V\left[G_{1}\right], \kappa_{1}=v^{+\omega^{n+1}}$, its successor is $\left(\kappa_{1}^{+\omega \cdot n+1}\right)^{V}$, and we have $\left(v^{+\alpha+1}, v^{+\alpha}\right) \rightarrow\left(v^{+\beta+1}, v^{+\beta}\right)$ for all limit ordinals 
$\omega \leqslant \beta<\alpha \leqslant \omega^{n+1}$. This is preserved by adjoining $\left\langle G_{j}: 2 \leqslant j<\omega\right\rangle$, which adds no subsets of $\left(\kappa_{1}^{+\omega \cdot n+1}\right)^{V}$. For $i>1$, we have that in $V\left[G_{i}\right]$,

$$
\left(\kappa_{i-1}^{+\omega \cdot n+\alpha+1}, \kappa_{i-1}^{+\omega \cdot n+\alpha}\right) \rightarrow\left(\kappa_{i-1}^{+\omega \cdot n+\beta+1}, \kappa_{i-1}^{+\omega \cdot n+\beta}\right)
$$

holds for all limit ordinals $0 \leqslant \beta<\alpha \leqslant \omega^{n+1}$. For each such $i$, these instances of Chang's Conjecture are preserved by adjoining $\left\langle G_{j}: i<j<\omega\right\rangle$, which adds no subsets of $\left(\kappa_{i}^{+\omega \cdot n+1}\right)^{V}$, the $\left(\omega^{n+1}+1\right)^{\text {st }}$ cardinal above $\kappa_{i-1}$ in the extension, and also by adjoining $G_{1} \times \cdots \times G_{i-1}$, which is generic for a $\kappa_{i-1}^{+\omega \cdot n}$-centered forcing. By the transitivity of Chang's Conjecture, we can combine finitely many instances to bridge the different intervals that lie between adjacent Prikry points, and get the weaker conclusion for $n+1$.

The hard part is to improve the final inequality to allow $\alpha=\omega^{n+2}$. If the critical point of $d$ as above is $\kappa$, then by applying transitivity again, it suffices to show that the extension satisfies $\left(\kappa^{+}, \kappa\right) \rightarrow\left(\kappa_{i}^{+}, \kappa_{i}\right)$ for infinitely many $i$. Towards this, we generalize Claim 3.11 and produce an iterated ultrapower for which we can find a generic filter for (the image of) a forcing $\mathbb{P} \in \mathrm{GS}_{n+1}$.

Claim 4.9 Suppose $1 \leqslant n<\omega$, W is a model of ZFC, and $\mathbb{P}(\mu, d) \in \mathrm{GS}_{n}^{W}$, with $\operatorname{crit}(d)=\kappa$. Suppose $p \in \mathbb{P}(\mu, d)$ is a condition of length $l, p=\left\langle f_{0}, \ldots, f_{l}\right\rangle \supset \vec{F}$. If $l>0$, let $v=\kappa_{l}^{+\omega \cdot n+2}$ and let $\mathbb{R}$ be such that $\mathbb{P}(\mu, d) \uparrow p \cong \mathbb{R} \times \mathbb{Q}$, as in Corollary 4.7. Otherwise let $v=\mu$ and let $\mathbb{R}$ be trivial.

There is an elementary embedding $j: W \rightarrow W^{\prime}$, where $W^{\prime}$ is transitive, $\operatorname{crit}(j)=$ $\kappa, j(\kappa)=\kappa^{+\omega \cdot n}$, and $\kappa^{+\omega \cdot n+1}$ is a fixed point of $j$. If there is a W-generic filter $H \subseteq \mathbb{R} \times \operatorname{Col}(\nu, \kappa)$, then there is a $W^{\prime}$-generic filter $G \subseteq j(\mathbb{P}(\mu, d))$ in $W[H]$ such that $j(p) \in G$. Moreover, $W[H]$ and $W^{\prime}[G]$ have the same $\kappa$-sequences of ordinals.

Proof First, let us introduce a temporary notation in order to describe generic filters for $\mathbb{P}(\mu, d)$. Every ordinal $\alpha<\omega^{\omega}$, can be represented using Cantor Normal Form as a sum

$$
\alpha=\omega^{m} \cdot k_{m}+\cdots+\omega \cdot k_{1}+k_{0},
$$

where $k_{i}<\omega$ for all $i$. For $\alpha \neq 0$, let $n_{\star}(\alpha)=\min \left\{r: k_{r} \neq 0\right\}$ and let $m_{\star}(\alpha)=k_{n_{\star}(\alpha)}$.

A generic $G \subseteq \mathbb{P}(\mu, d)$ can be unraveled into a sequence $\left\langle x_{\alpha}: 1 \leqslant \alpha<\omega^{n}\right\rangle \subseteq$ $\mathcal{P}_{\kappa}\left(H_{\kappa}+\omega \cdot n\right)$ and filters $\left\langle C_{\alpha}: \alpha<\omega^{n}\right\rangle$, from which we can recover $G$. If $\rho_{\alpha}=$ $x_{\alpha} \cap \kappa$, then the $\rho_{\alpha}$ are increasing, continuous, and cofinal in $\kappa . C_{0}$ is generic for $\operatorname{Col}\left(\mu, \rho_{1}\right)$, and for $\alpha \geqslant 1, C_{\alpha}$ is generic for $\operatorname{Col}\left(\rho_{\alpha}^{+\omega \cdot n_{\star}(\alpha)+\omega+2}, \rho_{\alpha+1}\right)$. If $\beta<\alpha$ and $n_{\star}(\beta) \leqslant n_{\star}(\alpha)$, then $x_{\beta} \in x_{\alpha}$.

Let us note that by unraveling the criteria for being in the filters associated to the sequences, we can recover $G$ in the following way. Let $\vec{F}=\left\langle F_{\alpha}: \alpha<\omega \cdot n\right\rangle$ be a sequence of functions. For each $\alpha<\omega^{n}$, define a finite sequence $\left\langle F_{\alpha}^{0}, \ldots, F_{\alpha}^{n-n_{\star}(\alpha)-1}\right\rangle$ by putting $F_{\alpha}^{0}=F_{\omega \cdot n_{\star}(\alpha)+m_{\star}(\alpha)}$, and for $0<k<n-n_{\star}(\alpha), F_{\alpha}^{k}=\pi\left(F_{\alpha}^{k-1}\right)$, where $\pi$ is the transitive collapse of $x_{\alpha+\omega^{n-k}}$, if that object is in dom $\pi$. Put $F_{\alpha}^{\prime}=F_{\alpha}^{n-n_{\star}(\alpha)-1}$. Then we have $\langle\varnothing\rangle \frown \vec{F} \in G$ iff for all $\alpha<\omega^{n}, F_{\alpha}^{\prime}$ is defined, $x_{\alpha} \in \operatorname{dom} F_{\alpha}^{\prime}$, and $F_{\alpha}^{\prime}\left(x_{\alpha}\right) \in C_{\alpha}$. 
Given a $\mathrm{GS}_{n}$-sequence $d$, let us construct an iterated ultrapower and a sequence $\left\langle x_{\alpha}, C_{\beta}: 1 \leqslant \alpha<\omega^{n}, \beta<\omega^{n}\right\rangle$ as above. We will assume, by induction on $n$ (simultaneously for all models of ZFC, all $\mathrm{GS}_{n}$-sequences $d$ and all generics $H$ ) that this process provides a generic filter for the limit ultrapower.

Let $\mu, d, H, W$ be as hypothesized, and let $d=\left\langle U_{\alpha}, K_{\alpha}: \alpha<\omega \cdot n\right\rangle$. Let us define by induction on $\omega^{n-1} \cdot l<\alpha \leqslant \omega^{n}$, a model $N_{\alpha}$ and elementary embeddings $j_{\beta, \alpha}: N_{\beta} \rightarrow N_{\alpha}$. The choice of the measures which are applied at each step resembles the iterated ultrapower for obtaining a Radin generic filter (see [18]).

Let $\alpha_{0}=\omega^{n-1} \cdot l+1$, and let $N_{\alpha_{0}}=W$. For limit ordinals $\alpha$, let $N_{\alpha}$ be the direct limit of the system $\left\langle N_{\beta}, j_{\beta, \gamma}: \beta<\gamma<\alpha\right\rangle$ and let $j_{\beta, \alpha}$ be the corresponding limit embeddings. For $\alpha=\beta+1$, let $j_{\beta, \alpha}: N_{\beta} \rightarrow N_{\alpha} \cong \operatorname{Ult}\left(N_{\beta}, j_{\alpha_{0}, \beta}\left(U_{\omega \cdot n_{\star}(\beta)+m_{\star}(\beta)}\right)\right)$, and let $j_{\gamma, \alpha}=j_{\beta, \alpha} \circ j_{\gamma, \beta}$ for $\gamma<\beta$. By Fact 3.10, $N_{\omega^{n}}$ is well-founded. By counting arguments similar to those in the previous section, we can show that $j_{\alpha_{0}, \omega^{n}}(\kappa)=\kappa^{+\omega \cdot n}$, and $j_{\alpha_{0}, \omega^{n}}\left(\kappa^{+\omega \cdot n+1}\right)=\kappa^{+\omega \cdot n+1}$.

Let us define a sequence of filters $\left\langle C_{i}: i<\omega^{n}\right\rangle$ and a sequence of sets $\left\langle x_{i}: 1 \leqslant i<\omega^{n}\right\rangle$. For $i \leqslant \omega^{n-1} \cdot l$ we extract $C_{i}$ and $x_{i}$ from the $W$-generic filter $H$.

Let us define the Prikry points for $\alpha>\omega^{n-1} \cdot l$. Let $X_{\alpha}=\kappa^{+m_{\star}(\alpha)}$ if $n_{\star}(\alpha)=0$,

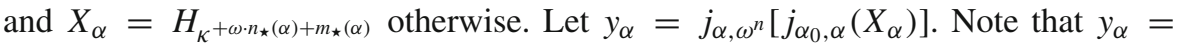
$j_{\alpha+1, \omega^{n}}\left(j_{\alpha, \alpha+1}\left[j_{\alpha_{0}, \alpha}\left(X_{\alpha}\right)\right]\right)$, and in particular it is in $N_{\omega^{n}}$. In other words, we take $y_{\alpha}$ to be the seed of the measure $j_{\alpha_{0}, \alpha}\left(U_{\omega \cdot n_{\star}(\alpha)+m_{\star}(\alpha)}\right)$, pushed by the map $j_{\alpha+1, \omega^{n}}$ to the limit model $N_{\omega^{n}}$. Since the critical point of the elementary map $j_{\alpha+1, \omega^{n}}$ is above the cardinality of $y_{\alpha}$, it acts pointwise.

If $n_{\star}(\alpha)=n-1$, let $x_{\alpha}=y_{\alpha}$. Otherwise, let $\pi$ be the Mostowski collapse of $y_{\alpha+\omega^{n_{\star}(\alpha)+1}}$ and let $x_{\alpha}=\pi\left(y_{\alpha}\right)$. Let $C_{\alpha}=j_{\alpha_{0}, \alpha}\left(K_{\omega \cdot n_{\star}(\alpha)+m_{\star}(\alpha)}\right)$. Let us verify that the obtained filter satisfies the requirements of Lemma 4.8 .

Let $m>l$. Let $G_{m}$ be the filter for the forcing $\mathbb{P}\left(\rho_{\omega^{n-1 \cdot(m-1)}}^{+\omega \cdot n+2}, d_{m}\right)^{N_{\omega^{n-1} \cdot m}}$, where $d_{m}=j_{\alpha_{0}, \omega^{n-1} \cdot m}(d)\lceil\omega \cdot(n-1)$, which is derived from the sequences $\left\langle x_{\alpha}: \omega^{n-1} \cdot(m-1) \leqslant \alpha<\omega^{n-1} \cdot m\right\rangle$ and $\left\langle C_{\alpha}: \omega^{n-1} \cdot(m-1) \leqslant \alpha<\omega^{n-1} \cdot m\right\rangle$. Let us assume, by induction, that $G_{m}$ is an $N_{\omega^{n-1} \cdot m}$-generic filter. Note that

$$
\mathbb{P}\left(\rho_{\omega^{n-1} \cdot(m-1)}^{+\omega \cdot n+2}, d_{m}\right)^{N_{\omega^{n-1} \cdot m}}=\mathbb{P}\left(\rho_{\omega^{n-1} \cdot(m-1)}^{+\omega \cdot n+2}, d_{m}\right)^{N_{\omega^{n}}}
$$

and that $G_{m}$ is also $N_{\omega^{n}}$-generic. For $m \leqslant l, G_{m}$ is derived from the $W$-generic filter $H$, and thus it is clearly $N_{\omega^{n}}$-generic.

Let $z_{i}=x_{\omega^{n-1} \cdot i}$ for $1 \leqslant i<\omega$. Let us check that for every sequence $\vec{F}=$ $\left\langle F_{i}: i<\omega \cdot n\right\rangle \in N_{\omega^{n}}$ there is some $k$ such that for all $m>k, \vec{F}\lceil\omega \cdot(n-1) \in$ $z_{m} \in \operatorname{dom} F_{\omega \cdot(n-1)+m}$, and $\left\langle F_{\omega \cdot(n-1)+m}\left(z_{m}\right)\right\rangle \sim_{m+1}\left(\vec{F}\lceil\omega \cdot(n-1)) \in G_{m+1}\right.$. Let us show that for $\alpha_{0} \leqslant \alpha<\omega^{n}$, if $\vec{F} \in N_{\alpha}$ is a sequence of functions such that $\langle\varnothing\rangle \frown \vec{F}$ is a condition in $j_{\alpha_{0}, \alpha}(\mathbb{P}(\mu, d))$, then for every $\beta>\alpha$,

$$
\begin{aligned}
& j_{\alpha, \omega^{n}}\left(\vec{F}\left\lceil\omega \cdot n_{\star}(\beta)\right) \in y_{\beta} \in \operatorname{dom} j_{\alpha, \omega^{n}}\left(F_{\omega \cdot n_{\star}}(\beta)+m_{\star}(\beta)\right),\right. \\
& \text { and } j_{\alpha, \omega^{n}}\left(F_{\omega \cdot n_{\star}(\beta)+m_{\star}(\beta)}\right)\left(y_{\beta}\right) \in C_{\beta} .
\end{aligned}
$$


The relation $j_{\alpha, \omega^{n}}\left(\vec{F}\left\lceil\omega \cdot n_{\star}(\beta)\right) \in y_{\beta}\right.$ holds simply because $\vec{F}\left\lceil\omega \cdot n_{\star}(\beta) \in\right.$ $\left(H_{j_{0}, \alpha}(\kappa)^{+\omega \cdot n_{\star}(\beta)+1}\right)^{N_{\alpha}}$. The other claims are true since $\bar{y}_{\beta}:=j_{\beta+1, \omega^{n}}^{-1}\left(y_{\beta}\right)$ is the seed of the measure $j_{\alpha_{0}, \beta}\left(U_{\omega \cdot n_{\star}(\beta)+m_{\star}(\beta)}\right)$ and the domain of $j_{\alpha, \beta}\left(F_{\omega \cdot n_{\star}(\beta)+m_{\star}(\beta)}\right)$ is large with respect to this measure. Moreover, this function represents an element of $j_{\alpha_{0}, \beta}\left(K_{\omega \cdot n_{\star}(\beta)+m_{\star}(\beta)}\right)$. But

$$
\begin{aligned}
j_{\alpha, \beta+1}\left(F_{\omega \cdot n_{\star}(\beta)+m_{\star}(\beta)}\right)\left(\bar{y}_{\beta}\right) & =j_{\beta, \beta+1}\left(j_{\alpha, \beta}\left(F_{\omega \cdot n_{\star}(\beta)+m_{\star}(\beta)}\right)\right)\left(\bar{y}_{\beta}\right) \\
& =\left[j_{\alpha, \beta}\left(F_{\omega \cdot n_{\star}}(\beta)+m_{\star}(\beta)\right)\right]_{j_{0}, \beta}\left(U_{\omega \cdot n_{\star}(\beta)+m_{\star}(\beta)}\right) \\
& \in j_{\alpha_{0}, \beta}\left(K_{\omega \cdot n_{\star}(\beta)+m_{\star}(\beta)}\right)=C_{\beta} .
\end{aligned}
$$

Note that for $\omega^{n-1} \cdot l<\alpha<\omega^{n}$, the sequence $\left\langle y_{\alpha}, y_{\alpha+\omega^{n}(\alpha)+1}, \ldots, y_{\alpha+\omega^{n-1}}\right\rangle$ is both $\in$ - and $\subseteq$-increasing. Thus to compute $x_{\alpha}$, we get the same result by taking the image of $y_{\alpha}$ under the transitive collapse $y_{\alpha+\omega^{n_{\star}(\alpha)+1}}$, as by first collapsing $y_{\alpha+\omega^{n-1}}$, then collapsing the image of $y_{\alpha+\omega^{n-2}}$, etc., until we take the image of $y_{\alpha}$ under $n-n_{\star}(\alpha)-1$ successive collapses. The point is that the latter process parallels exactly the sequence of collapses applied to a sequence of functions $\vec{F}$ to determine whether $\langle\varnothing\rangle \frown \vec{F}$ is in the filter generated from the sequences $\left\langle x_{\alpha}, C_{\beta}: 1 \leqslant \alpha<\omega^{n}, \beta<\omega^{n}\right\rangle$.

Hence, if

$$
\begin{aligned}
& j_{\alpha, \omega^{n}}\left(\vec{F}\left\lceil\omega \cdot n_{\star}(\beta)\right) \in y_{\beta} \in \operatorname{dom} j_{\alpha, \omega^{n}}\left(F_{\omega \cdot n_{\star}}(\beta)+m_{\star}(\beta)\right),\right. \\
& \text { and } j_{\alpha, \omega^{n}}\left(F_{\omega \cdot n_{\star}(\beta)+m_{\star}(\beta)}\right)\left(y_{\beta}\right) \in C_{\beta},
\end{aligned}
$$

then $j_{\alpha, \omega^{n}}(\vec{F})_{\beta}^{\prime} \in x_{\beta} \in \operatorname{dom} j_{\alpha, \omega^{n}}(\vec{F})_{\beta}^{\prime}$, and $j_{\alpha, \omega^{n}}(\vec{F})_{\beta}^{\prime}\left(x_{\beta}\right) \in C_{\beta}$. So if $\vec{F} \in N_{\alpha}$, the genericity criteria holds for $j_{\alpha, \omega^{n}}(\vec{F})$ for the cofinal segment above $\alpha$. Since $N_{\omega^{n}}$ is a direct limit, the generated filter $G$ is generic.

We would like to claim now that $N_{\omega^{n}}[G]$ has the same $\kappa$-sequences as $W[H]$. Indeed, since the forcing that introduces $H$ has cardinality $\kappa$, any sequence of ordinals in $W[H]$ has a name of cardinality $\kappa$ and thus can be coded using a sequence of ordinals of length $\kappa$ from $W$.

Let $\left\langle\xi_{i}: i<\kappa\right\rangle$ be a sequence of ordinals in $W$. In $N_{\omega^{n}}$, for every ordinal there is a representing function $f_{i}$, and a finite sequence $s_{i} \subseteq\left\langle y_{\alpha}: \omega^{n-1} \cdot l<\alpha<\omega^{n}\right\rangle$, such that $j_{\alpha_{0}, \omega^{n}}\left(f_{i}\right)\left(s_{i}\right)=\xi_{i}$. By our choices of $x_{i}$ and $C_{i}$, the sequence $\left\langle y_{\alpha}: \omega^{n-1} \cdot l<\alpha<\omega^{n}\right\rangle$ can be computed from the generic filter $G$. Since $j_{\alpha_{0}, \omega^{n}}\left(\left\langle f_{i}: i<\kappa\right\rangle\right)$ and $j_{\alpha_{0}, \omega^{n}}\left(\left\langle s_{i}: i<\kappa\right\rangle\right)$ are in $N_{\omega^{n}}$, and since $\left\langle y_{\alpha}: \omega^{n-1} \cdot l<\alpha<\omega^{n}\right\rangle \in N_{\omega^{n}}[G]$ we conclude that $\left\langle\xi_{i}: i<\kappa\right\rangle \in N_{\omega^{n}}[G]$.

Let us return to the proof of the theorem. Recall that, assuming the inductive claim holds for $\mathrm{GS}_{n}$, we must only show that for every $\mathbb{P}(\mu, d) \in \mathrm{GS}_{n+1}$ with crit $(d)=\kappa$, it is forced that $\left(\kappa^{+}, \kappa\right) \rightarrow\left(\kappa_{i}^{+}, \kappa_{i}\right)$ holds for infinitely many $i$. Let $p$ be a condition of length $l$, let $H \subseteq \mathbb{R} \times \operatorname{Col}(\nu, \kappa)$ be as in Claim 4.9, with $H$ generic over $V$. Note that $V[H] \models$ $\left|\left(\kappa_{l}^{+\omega \cdot n}\right)^{V}\right|=\kappa_{l}$, and $\left(\kappa_{l}^{+\omega \cdot n+1}\right)^{V}=\kappa_{l}^{+}$. By Lemma 4.3, $\left(\kappa^{+\omega \cdot(n+1)+1}, \kappa^{+\omega \cdot(n+1)}\right) \rightarrow$ $\left(\kappa_{l}^{+}, \kappa_{l}\right)$ holds in $V[H]$. Let $j: V \rightarrow M$ and $G$ be given by Claim 4.9, with $j(p) \in G$.

Let $\mathfrak{A} \in M[G]$ be any structure on $j\left(\kappa^{+\omega \cdot(n+1)+1}\right)=\kappa^{+\omega \cdot(n+1)+1}=\left(j(\kappa)^{+}\right)^{M[G]}$. By Chang's Conjecture in $V[H]$, there is a $\mathfrak{B} \prec \mathfrak{A}$ of size $\kappa_{l}^{+\omega \cdot n+1}$ such that 
$\left.\mid \mathfrak{B} \cap \kappa^{+\omega \cdot(n+1)}\right)|=| \mathfrak{B} \cap j(\kappa) \mid=\kappa_{l}^{+\omega \cdot n}$. By the closure of $M[G], \mathfrak{B} \in M[G]$, and thus $M[G] \models\left(j(\kappa)^{+}, j(\kappa)\right) \rightarrow\left(\kappa_{l}^{+}, \kappa_{l}\right)$. By elementarity, the desired conclusion follows.

\section{Chang's Conjecture with the same target}

In this section we will discuss two restricted versions of the Singular Global Chang's Conjecture.

Theorem 5.1 Suppose that $\kappa$ is $v^{+}$-supercompact, where $\operatorname{cf}(v)=\kappa^{+}$and $v$ is a limit of measurable cardinals, and $\alpha_{\star}$ is a countable ordinal. Then there is a generic extension in which

$$
\left(\mu^{+}, \mu\right) \rightarrow\left(\omega_{1}, \omega\right)
$$

for all $\mu<\aleph_{\alpha_{\star}}$.

Theorem 5.2 Suppose there are two supercompact cardinals and $\alpha_{\star}>0$ is a countable limit ordinal. Then there is a generic extension in which

$$
\left(\mu^{+}, \mu\right) \rightarrow\left(\aleph_{\alpha_{\star}+1}, \aleph_{\alpha_{\star}}\right),
$$

for all singular $\mu, \aleph_{\alpha_{\star}}<\mu<\aleph_{\omega_{1}}$.

The proof of both theorems follows closely the ideas from [13], which in turn are motivated by the forcing arguments from [17].

Proof of Theorem 5.1 Let us assume that $\kappa$ is Laver-indestructible (with respect to $\kappa$ directed closed forcing notions of cardinality $\leqslant v^{+}$) and that $\mathrm{GCH}$ holds above $\kappa$. If this is not the case, we can always force it using Laver forcing [16]. Let $\left\langle\zeta_{\beta}: \beta<\kappa^{+}\right\rangle$ be a continuous increasing sequence with $\sup \zeta_{\beta}=\nu, \zeta_{0}=\kappa$, and $\zeta_{\beta+1}$ measurable for each $\beta<\kappa^{+}$.

For every $\alpha<\kappa^{+}$of countable cofinality, let us pick an increasing cofinal $\omega$ sequence $s_{\alpha}: \omega \rightarrow \alpha$. Let us assume that for each $\alpha, s_{\alpha}(0)=0$, and $s(n)$ is a successor ordinal for $n>0$.

Let us consider the forcing

$$
\mathbb{C}_{\alpha}=\prod_{n<\omega} \mathbb{E}\left(\zeta_{s_{\alpha}(n)}, \zeta_{s_{\alpha}(n+1)}\right) \times \operatorname{Col}\left(\zeta_{\alpha}^{+}, v^{+}\right)
$$

where $\mathbb{E}(\mu, \delta)$ is the Easton-support product of $\operatorname{Col}(\mu, \eta)$ over all inaccessible $\eta<\delta$. The product in the definition of $\mathbb{C}_{\alpha}$ is taken with full support. For properties of the Easton collapse, see [24].

For each $\alpha<\kappa^{+}$of countable cofinality, after forcing with $\mathbb{C}_{\alpha}$,

$$
\left(\zeta_{\alpha}^{+}\right)^{V}=\left(\kappa^{+\omega+1}\right)^{V^{\mathbb{C}_{\alpha}}}
$$


By the arguments of [6] related to Lemma 3.1, there is $\rho_{\alpha}<\kappa$ such that

$$
V^{\mathbb{C}_{\alpha}} \models\left(\kappa^{+\omega+1}, \kappa^{+\omega}\right) \rightarrow\left(\rho_{\alpha}^{+}, \rho_{\alpha}\right),
$$

and this remains true after forcing with $\mathbb{D}_{\alpha}=\operatorname{Col}\left(\omega, \rho_{\alpha}\right) * \operatorname{Col}\left(\rho_{\alpha}^{+},<\kappa\right)$. In fact, $\left(\zeta_{\alpha}^{+}, \zeta_{\alpha}\right) \rightarrow\left(\rho_{\alpha}^{+}, \rho_{\alpha}\right)$ must already hold in $V$, by the distributivity of $\mathbb{C}_{\alpha}$.

Since the forcing $\mathbb{C}_{\alpha}$ is weakly homogeneous, the value of $\rho_{\alpha}$ depends only on $\alpha$ and does not depend on the generic filter for $\mathbb{C}_{\alpha}$. Therefore, the function $\alpha \mapsto \rho_{\alpha}$ belongs to the ground model, $V$, and has the property that

$$
1 \Vdash \mathbb{D}_{\alpha} \times \mathbb{C}_{\alpha}\left(\kappa^{+\omega+1}, \kappa^{+\omega}\right) \rightarrow\left(\check{\rho}_{\alpha}^{+}, \check{\rho}_{\alpha}\right) .
$$

By the $\kappa^{+}$-completeness of $\mathrm{NS}_{\kappa^{+}}$, there is a stationary set $S \subseteq \kappa^{+}$and a cardinal $\rho_{\star}<\kappa$ such that for all $\alpha \in S, \rho_{\alpha}=\rho_{\star}$. Let $\mathbb{D}$ be the common value of $\mathbb{D}_{\alpha}$ for $\alpha \in S$. There is $n_{0}<\omega$ such that for every club $C \subseteq \kappa^{+},\left\{s_{\alpha}\left(n_{0}\right): \alpha \in C \cap S\right\}$ is unbounded. By Fodor's Lemma, we may assume that $s_{\alpha}\left\lceil n_{0}\right.$ is constant on $S$.

Let us define a partial order $\mathbb{P}$ that searches for a "thread" of the sequences $s_{\alpha}$ for $\alpha \in S$. A condition $t \in \mathbb{P}$ is a continuous increasing function from a countable successor ordinal $\gamma$ into $\kappa^{+}$, such that $\operatorname{ran}(t) \subseteq S \cup \bigcup_{\alpha<\kappa^{+}} \operatorname{ran}\left(s_{\alpha}\right)$, and for every limit ordinal $\beta<\gamma, \operatorname{ran}\left(s_{t(\beta)}\right) \subseteq \operatorname{ran}(t)$. As in [13], we have:

Claim 5.3 For every $t \in \mathbb{P}$, every $\gamma<\omega_{1}$, and every $\xi<\kappa^{+}$, there is a stronger condition $t^{\prime} \supseteq t$ with $\gamma \subseteq \operatorname{dom} t^{\prime}$ and $s_{\beta}\left(n_{0}\right)>\xi$ for limit $\beta \in \operatorname{dom} t^{\prime} \backslash \operatorname{dom} t$.

In particular, we can find a thread of any countable length. Let $t$ be a thread of length $\alpha_{\star}$. Define a sequence $s: \alpha_{\star} \rightarrow v$ as follows. If $\beta$ is an infinite limit ordinal, then $s(\beta)=\zeta_{t(\beta)}^{+}$, and otherwise $s(\beta)=\zeta_{t(\beta)}$. Consider the forcing

$$
\mathbb{C}=\prod_{\beta<\alpha_{\star}} \mathbb{E}(s(\beta), s(\beta+1)) .
$$

First let us claim that in the generic extension by $\mathbb{D} \times \mathbb{C}$, we have $\left(\aleph_{\beta+1}, \aleph_{\beta}\right) \rightarrow$ $\left(\aleph_{1}, \aleph_{0}\right)$ for limit $\beta<\alpha_{\star}$. As in [13], the projection properties of the Levy collapse, together with the fact that $\operatorname{ran}\left(s_{\beta}\right) \subseteq \operatorname{ran}(t)$ for limit $\beta<\alpha_{\star}$, imply that for each limit $\beta<\alpha_{\star}$, there is a projection $\pi_{\beta}: \mathbb{C}_{\beta} \rightarrow \mathbb{C}$. If $\mathfrak{A}$ is a structure on $\zeta_{\beta}^{+}$in $V^{\mathbb{D} \times \mathbb{C}}$, then in $V^{\mathbb{D} \times \mathbb{C}_{\beta}}$, there is an elementary $\mathfrak{B} \prec \mathfrak{A}$ such that $|\mathfrak{B}|=\rho_{\star}^{+}=\aleph_{1}$, and $\left|\mathfrak{B} \cap \zeta_{\beta}\right|=\left|\rho_{\star}\right|=\aleph_{0}$. Since the quotient forcing adds no sets of ordinals of size $<\kappa=\aleph_{2}$, the instance of Chang's Conjecture holds in $V^{\mathbb{D} \times \mathbb{C}}$.

To obtain the result for successors below $\alpha_{\star}$, we consider instead the forcing $\mathbb{D} * \dot{\mathbb{C}}$, where $\dot{\mathbb{C}}$ is the forcing with the same definition as $\mathbb{C}$, but constructed in $V^{\mathbb{D}}$ rather than $V$. By [23], there is a projection from $\mathbb{D} \times \mathbb{C}$ to $\mathbb{D} * \dot{\mathbb{C}}$ that is the identity on $\mathbb{D}$. By the same argument as above, the relevant instances of Chang's Conjecture at limit ordinals also hold in $V^{\mathbb{D} * \dot{\mathbb{C}}}$.

Suppose $\beta<\alpha_{\star}$ is zero or a successor ordinal. Let $\zeta=s(\beta)=\zeta_{t(\beta)}$, and let $\eta$ be the predecessor of $\zeta$ in the extension by $\mathbb{D} * \dot{\mathbb{C}}$, which is regular. Since $\zeta$ is measurable, 
in the extension

$$
\mathbb{D} * \prod_{\gamma<\beta} \dot{\mathbb{E}}(s(\gamma), s(\gamma+1))
$$

there is a normal ideal $I$ on $\zeta$ such that $\mathcal{P}(\zeta) / I$ contains a countably closed dense setin particular the boolean algebra is a proper forcing. By [20], the following version of Strong Chang's Conjecture holds in this model: If $M$ is a countable elementary submodel of $H_{\zeta^{+}}$then there is an elementary $M^{\prime} \supseteq M$ such that $M \cap \eta=M^{\prime} \cap \eta$ and $M \cap \zeta \neq M^{\prime} \cap \zeta$. By [6, Lemma 15], $(\zeta, \eta) \rightarrow\left(\aleph_{1}, \aleph_{0}\right)$ is preserved by the formerly $\zeta$-closed quotient $\prod_{\beta \leqslant \gamma<\alpha_{\star}} \mathbb{E}(s(\gamma), s(\gamma+1))$.

Remark 5.4 Note that the assumption that $v$ is a limit of measurable cardinals is used in order to get Chang's Conjecture between successors of regulars and $\omega_{1}$. If we only want Chang's Conjecture to hold between successors of singulars and $\omega_{1}$, we can drop this assumption.

Proof of Theorem 5.2 Let $\kappa_{0}<\kappa$ be supercompact, and let $\alpha_{\star}>0$ be a fixed countable limit ordinal. First force Martin's Maximum (MM) while turning $\kappa_{0}$ into $\aleph_{2}$, as in [10]. By [15], MM is indestructible under $\aleph_{2}$-directed-closed forcing. Then, force with Laver's forcing, which is $\aleph_{2}$-directed-closed, to force that $\kappa$ is indestructibly supercompact and GCH holds above $\kappa$.

Next we need, for large enough $\mu<\kappa$, a forcing $\mathbb{D}_{\mu}$ that turns $\kappa$ into $\aleph_{\alpha_{\star}+3}$ while preserving $\omega_{1}$ and satisfying the hypotheses of Lemma 3.1. If $\tau\left(\alpha_{\star}\right)=\omega$, let $\mathbb{D}_{\mu}=\operatorname{Col}\left(\aleph_{\iota\left(\alpha_{\star}\right)+1}, \mu\right) \times \operatorname{Col}\left(\mu^{+\omega+2},<\kappa\right)$. If $\tau\left(\alpha_{\star}\right)>\omega$, let $\vec{\gamma}$ be the identity sequence converging to $\omega$, and let $\vec{\delta}$ be a non-decreasing sequence summing to $\tau\left(\alpha_{\star}\right)$, with $\delta_{1} \geqslant \omega$. Let $\mathbb{D}_{\mu}=\mathbb{P}\left(\aleph_{\iota\left(\alpha_{\star}\right)+1}, \vec{\gamma}, \vec{\delta}, \vec{U}, \vec{K}\right) \times \operatorname{Col}\left(\mu^{+\omega+2},<\kappa\right)$, where $\vec{U}$ and $\vec{K}$ are $\omega$-sequences such that $U_{n}$ is a normal $\mu$-complete ultrafilter on $\mathcal{P}_{\mu}\left(\mu^{+n}\right)$, and $K_{n}$ is sufficiently generic filter, as in Sect. 3.

Working in a model of MM, let us repeat the arguments from the beginning of the proof of Theorem 5.1. For each $\alpha<\kappa^{+}$of countable cofinality, choose a cofinal increasing sequence $s_{\alpha}: \omega \rightarrow \alpha$ with $s_{\alpha}(0)=\kappa$ and $s_{\alpha}(n)$ is a double successor ordinal for $n>0$. For each $\alpha<\kappa^{+}$of countable cofinality, define

$$
\mathbb{C}_{\alpha}=\prod_{n<\omega} \operatorname{Col}\left(\kappa^{+s_{\alpha}(n)}, \kappa^{+s_{\alpha}(n+1)-1}\right) \times \operatorname{Col}\left(\kappa^{+\alpha+2}, \kappa^{+\kappa^{+}+1}\right) .
$$

For each $\alpha$, there is $\mu_{\alpha}<\kappa$ such that

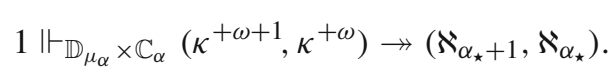

As above, let $S \subseteq \kappa^{+}$be a stationary set of countable cofinality ordinals such that $\mu_{\alpha}$ has the same value for all $\alpha \in S$, and that the threading forcing $\mathbb{P}$ satisfies Claim 5.3. In particular, there is $n_{0}<\omega$ such that for all club $C \subseteq \kappa^{+},\left\{s_{\alpha}\left(n_{0}\right): \alpha \in S \cap C\right\}$ is unbounded, and $s_{\alpha}\left\lceil n_{0}\right.$ is the same for all $\alpha \in S$. Let $\mathbb{D}=\mathbb{D}_{\mu_{\alpha}}$ for any $\alpha \in S$. We now claim that $\mathbb{P}$ preserves stationary subsets of $\omega_{1}$. This is a reminiscent of the forcing for Friedman's Problem (see [10, Theorem 9]). 
Fix a stationary set $A \subseteq \omega_{1}$ and a condition $t_{0} \in \mathbb{P}$. Let $\dot{C}$ be a $\mathbb{P}$-name for a club subset of $\omega_{1}$, and let

$$
M \prec\left(H_{\kappa^{++}}, \in,\left\langle s_{\alpha}: \alpha<\kappa^{+}\right\rangle, S, \mathbb{P}, t_{0}, A, \dot{C}, \triangleleft\right)
$$

be such that $M \cap \kappa^{+}=\delta \in S$, where $\triangleleft$ is a well-order of $H_{\kappa^{++}}$. Let us assume further that $M$ is the union of an increasing sequence of models $M_{n}$ such that $M_{n} \in M_{n+1}$. We may also assume that $s_{\delta}\left(n_{0}\right)>\sup \left(M_{0} \cap \delta\right)$.

Let $N_{n}^{\prime} \prec M_{n}$ be the Skolem hull of the finite set $\operatorname{ran}\left(s_{\delta}\right) \cap M_{n}$. For $\alpha<\omega_{1}$ and $n<\omega$, let $N_{n}^{\prime}[\alpha]$ be the Skolem hull of $N_{n}^{\prime} \cup \alpha$. There is some $\alpha<\omega_{1}$ such that for all $n<\omega, N_{n}^{\prime}[\alpha] \cap \omega_{1}=\alpha \in A$. Let $N_{n}=N_{n}^{\prime}[\alpha]$ for such an $\alpha$. Let $N=\bigcup N_{n}$, so $N \prec M$ is countable, $\sup \left(N \cap \kappa^{+}\right)=\delta, \operatorname{ran}\left(s_{\delta}\right) \subseteq N$, and $N \cap \omega_{1} \in A$.

Let $\left\langle D_{n}: n<\omega\right\rangle$ enumerate the dense subsets of $\mathbb{P}$ in $N$, such that $D_{n} \in N_{n}$. Using Claim 5.3, we can build a sequence $t_{0} \geqslant t_{1} \geqslant t_{2} \geqslant \cdots$ such that for $n>0, t_{n} \in D_{n} \cap N_{n}$ and $\operatorname{ran}\left(s_{\delta}\right) \cap N_{n} \subseteq \operatorname{ran}\left(t_{n}\right)$. We achieve that by working inside $N_{n}$. We first extend $t_{n-1}$ by the finite set $\operatorname{ran}\left(s_{\delta}\right) \cap N_{n}$ and then extend this condition to meet $D_{n}$. Let $\gamma=\operatorname{ot}\left(\bigcup_{n} t_{n}\right)$, and let $t=\bigcup_{n} t_{n} \cup\{\langle\gamma, \delta\rangle\}$. Then $t$ is an $(N, \mathbb{P})$-master condition, and so it forces $A \cap \dot{C} \neq \varnothing$.

Applying MM, we find a thread $t$ of length $\omega_{1}$. Let $s: \omega_{1} \rightarrow \kappa^{+}$be such that $s(\alpha)=t(\alpha)+2$ for limit $\alpha>0$ and $s(\alpha)=t(\alpha)$ otherwise. Let us consider the forcing

$$
\mathbb{C}=\prod_{\alpha<\omega_{1}} \operatorname{Col}\left(\kappa^{+s(\alpha)}, \kappa^{+s(\alpha+1)-1}\right)
$$

For every $\beta \in S$, there is a projection from $\mathbb{C}_{\beta}$ to $\mathbb{C}$. Therefore, since the quotient adds no sets of ordinals of size $<\kappa, \mathbb{D} \times \mathbb{C}$ forces the desired conclusion.

Remark 5.5 By slightly modifying the proof of Theorem 5.2, one can strengthen the conclusion of the theorem as follows. Suppose MM holds and there is a supercompact cardinal. For every $\beta<\omega_{2}$ and every nonzero $\alpha_{\star}<\beta$ of countable cofinality, there is an $\omega_{1}$-preserving generic extension in which $\left(\mu^{+}, \mu\right) \rightarrow\left(\aleph_{\alpha_{\star}+1}, \aleph_{\alpha_{\star}}\right)$ for all $\mu<\aleph_{\beta}$, such that $\operatorname{cf} \mu=\omega$ and $\mu>\aleph_{\alpha_{\star}}$.

\section{Open problems}

The construction in Sect. 4 is limited to instances of Chang's Conjecture between successors of singular cardinals below $\aleph_{\omega^{\omega}}$. In order to push this mechanism forwards, one needs to start with a model in which there is a cardinal $\kappa$ which is $\kappa^{+\alpha+1}$-supercompact and Chang's Conjecture holds between any pair of singular cardinals in the interval $\left[\kappa, \kappa^{+\alpha}\right]$. Since our method to produce an interval with such properties with limits of limit cardinals includes Prikry forcing, it cannot preserve supercompactness.

Question 6.1 Is it consistent relative to large cardinals that $\left(\mu^{+}, \mu\right) \rightarrow\left(v^{+}, v\right)$ holds whenever $\mu$ and $v$ have countable cofinality? 
The known limitations on Global Chang's Conjecture do not seem to rule out the consistency of a strengthening of Theorem 5.1 to a global statement:

Question 6.2 Is it consistent relative to large cardinals that $\left(\kappa^{+}, \kappa\right) \rightarrow\left(\omega_{1}, \omega\right)$ holds for all infinite cardinals $\kappa$ ?

Funding Open access funding provided by University of Vienna.

Open Access This article is licensed under a Creative Commons Attribution 4.0 International License, which permits use, sharing, adaptation, distribution and reproduction in any medium or format, as long as you give appropriate credit to the original author(s) and the source, provide a link to the Creative Commons licence, and indicate if changes were made. The images or other third party material in this article are included in the article's Creative Commons licence, unless indicated otherwise in a credit line to the material. If material is not included in the article's Creative Commons licence and your intended use is not permitted by statutory regulation or exceeds the permitted use, you will need to obtain permission directly from the copyright holder. To view a copy of this licence, visit http://creativecommons.org/licenses/by/4.0/.

\section{References}

1. Abraham, U., Magidor, M.: Cardinal arithmetic. In: Foreman, M., Kanamori, A. (eds.) Handbook of Set Theory. Vols. 1, 2, 3, pp. 1149-1227. Springer, Dordrecht (2010)

2. Ben-Neria, O., Lambie-Hanson, C., Unger, S.: Diagonal supercompact Radin forcing. Ann. Pure Appl. Log. 171(10), \# 102828 (2020)

3. Bukovský, L.: Iterated ultrapower and Prikry's forcing. Comment. Math. Univ. Carolinae 18(1), 77-85 (1977)

4. Cummings, J., Foreman, M., Magidor, M.: Squares, scales and stationary reflection. J. Math. Log. 1(1), 35-98 (2001)

5. Dehornoy, P.: Iterated ultrapowers and Prikry forcing. Ann. Math. Logic 15(2), 109-160 (1978)

6. Eskew, M., Hayut, Y.: On the consistency of local and global versions of Chang's conjecture. Trans. Amer. Math. Soc. 370(4), 2879-2905 (2018)

7. Foreman, M.: Smoke and mirrors: combinatorial properties of small cardinals equiconsistent with huge cardinals. Adv. Math. 222(2), 565-595 (2009)

8. Foreman, M., Magidor, M.: Large cardinals and definable counterexamples to the continuum hypothesis. Ann. Pure Appl. Logic 76(1), 47-97 (1995)

9. Foreman, M., Magidor, M.: A very weak square principle. J. Symbolic Logic 62(1), 175-196 (1997)

10. Foreman, M., Magidor, M., Shelah, S.: Martin's maximum, saturated ideals, and nonregular ultrafilters. I. Ann. Math. 127(1), 1-47 (1988)

11. Gitik, M.: Prikry-type forcings. In: Foreman, M., Kanamori, A. (eds.) Handbook of Set Theory. Vols. 1, 2, 3, pp. 1351-1447. Springer, Dordrecht (2010)

12. Gitik, M., Sharon, A.: On SCH and the approachability property. Proc. Amer. Math. Soc. 136(1), $311-320$ (2008)

13. Golshani, M., Hayut, Y.: The tree property on a countable segment of successors of singular cardinals. Fund. Math. 240(2), 199-204 (2018)

14. Krueger, J.: Radin forcing and its iterations. Arch. Math. Logic 46(3-4), 223-252 (2007)

15. Larson, P.: Separating stationary reflection principles. J. Symbolic Logic 65(1), 247-258 (2000)

16. Laver, R.: Making the supercompactness of $\kappa$ indestructible under $\kappa$-directed closed forcing. Israel J. Math. 29(4), 385-388 (1978)

17. Magidor, M., Shelah, S.: When does almost free imply free? (For groups, transversals, etc.). J. Amer. Math. Soc. 7(4), 769-830 (1994)

18. Mitchell, W.J.: Beginning inner model theory. In: Foreman, M., Kanamori, A. (eds.) Handbook of Set Theory. Vols. 1, 2, 3, pp. 1449-1495. Springer, Dordrecht (2010)

19. Perlmutter, N.L.: The large cardinals between supercompact and almost-huge. Arch. Math. Logic 54(3-4), 257-289 (2015)

20. Sakai, H.: Semiproper ideals. Fund. Math. 186(3), 251-267 (2005) 
21. Shelah, S.: Reflecting stationary sets and successors of singular cardinals. Arch. Math. Logic 31(1), 25-53 (1991)

22. Shelah, S.: Proper and Improper Forcing. Perspectives in Mathematical Logic, 2nd edn. Springer, Berlin (1998)

23. Shioya, M.: The Easton collapse and a saturated filter. RIMS Kokyuroku 1754, 108-114 (2011)

24. Shioya, M.: Easton collapses and a strongly saturated filter. Arch. Math. Logic 59(7-8), 1027-1036 (2020)

25. Steel, J.: An introduction to iterated ultrapowers. In: Chong, C., et al. (eds.) Forcing, Iterated Ultrapowers, and Turing Degrees. Lecture Notes Series. Institute for Mathematical Sciences. National University of Singapore, vol. 29, pp. 123-174. World Scientific, Hackensack (2016)

Publisher's Note Springer Nature remains neutral with regard to jurisdictional claims in published maps and institutional affiliations. 